\title{
Data-Driven Optimal Charging Decision Making for Connected and Automated Electric Vehicles: A Personal Usage Scenario
}

Zonggen Yi, Matthew Shirk November 2017

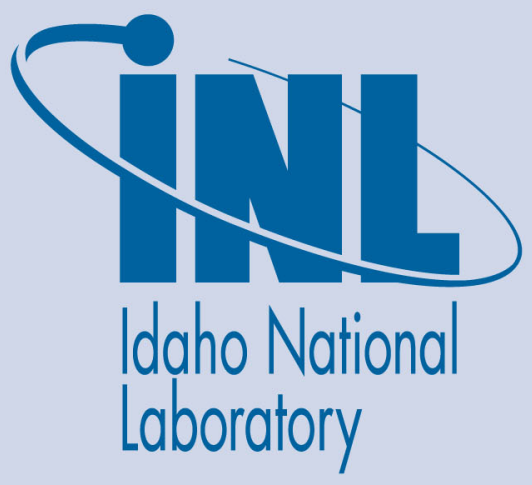

The INL is a U.S. Department of Energy National Laboratory operated by Battelle Energy Alliance 


\title{
Data-Driven Optimal Charging Decision Making for Connected and Automated Electric Vehicles: A Personal Usage Scenario
}

\author{
Zonggen Yi, Matthew Shirk
}

November 2017

Idaho National Laboratory Idaho Falls, Idaho 83415

http://www.inl.gov

Prepared for the U.S. Department of Energy

Office of Energy Efficiency and Renewable Energy Under DOE Idaho Operations Office Contract DE-AC07-05ID14517 


\title{
Data-Driven Optimal Charging Decision Making for Connected and Automated Electric Vehicles: A Personal Usage Scenario
}

\author{
Zonggen $\mathrm{Yi}^{*}$ \\ Matthew Shirk \\ Idaho National Laboratory \\ 2525, Fremont Drive, Idaho Falls, ID, USA
}

\begin{abstract}
This study introduces an optimal charging decision making framework for connected and automated electric vehicles under a personal usage scenario. This framework aims to provide charging strategies, i.e. the choice of charging station and the amount of charged energy, by considering constraints from personal daily itineraries and existing charging infrastructure. A data-driven method is introduced to establish a stochastic energy consumption prediction model with consideration of realistic uncertainties. This is performed by analyzing a large scale electric vehicle data set. A real-time updating method is designed to construct this prediction model from new consecutive data points in an adaptive way for real-world applications. Based on this energy cost prediction framework from real electric vehicle data, multistage optimal charging decision making models are introduced, including a deterministic model for average outcome decision making and a robust model for safest charging strategies. A dynamic programming algorithm is proposed to find the optimal charging strategies. Detailed simulations and case studies demonstrate the performance of the proposed algorithms to find optimal charging strategies. They also show the potential capability of connected and automated electric vehicles to reduce the range anxiety and charging infrastructure dependency.
\end{abstract}

Key words:

Data-Driven Method, Charging Decision Making, Connected and Automated Electric Vehicles, Energy Consumption Prediction, Multistage Decision Making, Dynamic Programming

\footnotetext{
* Corresponding author.

Email addresses: Zonggen.Yi@inl.gov (Zonggen Yi)
}

Preprint submitted to Transportation Research Part C: Emerging TechnologiesOctober 24, 2017 


\section{Introduction}

Electrification of transportation by increasing electric vehicle (EV) usage has important impacts on greenhouse gas emissions and energy dependency (Sioshansi and Denholm (2009); Eberle and Von Helmolt (2010); Armaroli and Balzani (2011)). Tremendous work is being performed to electrify powertrain systems and the transportation system (Bilgin et al. (2015)). Accelerating EV adoption may be a key strategy for helping regions achieve national- and statelevel transportation sustainability. Besides great progress in electric drive systems, recently most automakers and some high-tech companies, e.g. Google, Uber, etc., are focusing on implementing autonomous driving technology. They are trying to put forward the real-world application of this technology. Furthermore, the automotive OEMs are combining autonomous driving technology with electric vehicles. For example, all Tesla cars being produced now have full self-driving hardware (Tesla (2016)). General Motors is also testing the autonomous driving on its new Chevrolet Bolt (electrek (2017)). Only a few of them are named here. Car-sharing or car-hailing companies plan to use both electric vehicles and autonomous driving in their transportation network. Selfdriving technology is an important aspect to improve their service quality and reduce operation costs. Electrified vehicles can help to improve the energy efficiency. These two trends will work together to improve the intelligence and sustainability of transportation system in the coming future.

Current electric vehicles still have the essential barrier of long charging time compared to conventional vehicles. Relative high costs are necessary for satisfying charging requirements. For example, the construction cost of charging stations, the long charging time for EV user, etc.. However, the introduction of autonomous driving technology would remove the challenge of co-locating charging infrastructure with driver destinations and presents a driver-free method for EVs to reach nearby charging stations. This will significantly change the charging behavior of electric vehicles. EV driver will no longer need to be present at charging stations for charging actions. Automated EVs can drive to nearby charging stations to perform charging actions by themselves when necessary. Meanwhile, connected vehicles technology is emerging to make real-time connections between vehicles and infrastructure networks. Electric vehicles will have the capability to sense and obtain pertinent information from nearby charging station networks and then calculate the corresponding costs and availability for charging. This information will be very helpful for real-time, optimal and sustainable charging decision-making for electric vehicles. It has to say that autonomous vehicle technology cannot solve all the city transportation problem as discussed in UITP (2017). However, it has huge potential to improve the convenience and sustainability of EV charging actions.

This study attempts to establish an optimal and sustainable charging decisionmaking framework for connected and automated electric vehicles (CAEVs). The personal usage scenario of CAEVs with time and distance constraints of daily itineraries is the main focus in this paper. The objective of this framework aims to design optimal charging strategies for minimum charging cost (e.g. monetary 
cost) outside home and also minimization of travel or energy cost attributed to charging actions. More details will be introduced in the following sections. This paper is organized as follows: Section 2 provides the related previous research and also our contributions in this paper. Section 3 introduces a multi-channel stochastic energy consumption prediction model and the corresponding realtime updating algorithm based on the data-driven method. Based on the energy cost prediction model, multistage optimal charging decision-making models are proposed in Section 4. Section 5 proposes a dynamic programming procedure for optimal charging strategies. Simulations and case studies in Section 6 are used to demonstrate the introduced framework. Section 7 concludes our work in this paper and also discusses some potential future research.

\section{Previous Research and Our Contributions}

Charging decision making for electric vehicles includes several aspects, e.g. the deployment of charging infrastructure, the analysis of charging behavior and the design of charging strategies. A lot of research has been performed for charging station deployment and charging behavior analysis. Different methods have been introduced for charging station deployment according to specific requirements and realistic situations, for example, deployment of both stationary and dynamic charging infrastructure for electric vehicles along traffic corridors in Chen et al. (2017b), data-driven method for siting and sizing of electric taxi charging stations in Yang et al. (2017), charging station deployment on urban road network in He et al. (2015, 2016); Giménez-Gaydou et al. (2016); Frade et al. (2011); Yi and Bauer (2016a), activity-based approach in Dong et al. (2014), and many more, e.g. Xylia et al. (2017); Tu et al. (2016); Ghamami et al. (2016); Li et al. (2016), etc. Deployment methods of wireless charging facilities are also being investigated in current literature, for example, Chen et al. (2017b), Deflorio and Castello (2017), Fuller (2016), Riemann et al. (2015). For charging behavior analysis, two multinomial logit-based and two nested logitbased models are proposed in Yang et al. (2016) for modeling the charging and route choice behavior of BEV drivers. The behaviors of electric vehicle driver and parking pattern are analyzed in (Marmaras et al. (2017); Latinopoulos et al. (2017); Birrell et al. (2015)). In contrast to a large body of literature on charging station deployment and charging behavior analysis, studies looking at the design of charging strategies of electric vehicles in a connected and fully automated setting are more limited.

The topic of optimal charging decision making in this paper focus on the design of charging strategies. It is also part of smart charging, which improves the interactions among the smart grid and EVs. Smart charging has been investigated thoroughly in previous research from different aspects. Most of them focused on the interaction with the power grid, for example, the smart energy supply (Wang et al. (2016)) and the impact of charging action on the power grid (Sundstrom and Binding (2012); Hu et al. (2014)). However, smart charging management from the viewpoint of the electric vehicle is also a very important aspect for EV users. The problem studied in this paper can be 
considered as trip level charging management for travel demand. An intelligent and sustainable way to charge is crucial to improve the driving experience and reduce the range anxiety of electric vehicle users. But there is little research about this topic. Most topics focused on the energy demand estimation (Bae and Kwasinski (2012); Yi and Bauer (2016b)) or existing charging behavior analysis (Marmaras et al. (2017); Latinopoulos et al. (2017); Yang et al. (2016); Birrell et al. (2015); Smart and Schey (2012)). This is because most current charging decisions are made by EV owners according to their experiences and driving demand. A charging decision-making system for current personal EV usage is not as important as other problems. There is some research on charging decision making in the fleet management system, for example the work in (Chen et al. (2016a), Pourazarm et al. (2016)). Charging strategy is a relatively more important aspect in EV fleet operation than for individual users. It aims to make sure an EV fleet can always have enough energy to perform services. However, this research doesn't investigate decision making by involving the autonomous driving setting. And there are different requirements for personal and fleet vehicle usage.

With the emerging of autonomous driving and its application in electric vehicles, the charging decision making should be taken over by the vehicles. Autonomous electric vehicles will require the capability to make charging decisions according to the battery energy state, the travel demand and also the available charging infrastructure. Automated electric vehicle charging stations will become available in the future (Corbett and Maniaci (2013); Tesla (2017)). Some works have touched this topic under the car-sharing situation. The work in Fagnant and Kockelman (2014) describes the design of an agent-based model for shared autonomous vehicle (SAV) operations. Chen et al. (2016b) further explores the management of a fleet of shared autonomous electric vehicles (SAEVs) in a regional, discrete-time, agent-based model. Although charging actions have been touched in this paper, the discussion for charging decision making is relatively initial. None of this previous work investigates the charging decision making for the connected and automated electric vehicles under a personal usage scenario. Due to the fully self-driving and self-charging capability assumption, electric vehicles can be charged at locations that are near the visited locations. Optimal charging strategies can help CAEVs to manage the charging actions along the route in order to make sure future itineraries can be completed with enough energy.

In order to solve this trip-level charging decision making problem, a prediction model of energy demand in coming trips is necessary. Previous methods studied energy cost prediction from two main aspects: One aspect is the physical model method, for example, tractive effort models (Prins et al. (2013)), powerbased energy consumption models (Yi and Bauer (2017a, 2016b); Fiori et al. (2016)) and energy consumption model based on generic high-level specifications and technical characteristics (Genikomsakis and Mitrentsis (2017)). The other aspect comprises data analysis methods, for example, feature-based linear regression from historical driving data (Ondruska and Posner (2014)), a systematic energy consumption estimation approach based on driving condi- 
tions (Zhang and Yao (2015)), and multiple linear regression methods based on real-world data (De Cauwer et al. (2015); Chen et al. (2017a)). However, physical models need high-resolution real time information to support accurate predictions. Data analysis methods in the literature don't involve inner physical relationships between the energy cost and traffic conditions. To our best knowledge, previous methods didn't model the uncertainties of energy cost. This should be an important aspect under different traffic and environmental conditions in a realistic world. The consideration of uncertainties is very crucial for robust charging strategies.

The main objective of this research is to introduce an optimal charging decision making framework for CAEVs under a personal usage scenario. This framework aims to provide charging strategies, i.e. the optimal choice of charging station outside home and the amount of charged energy, by considering constraints from personal daily itineraries and existing charging infrastructure. Abundant real world EV data is utilized to construct stochastic energy consumption prediction model, which is an important and necessary part of the charging decision making framework. The proposed framework is a dynamic and multistage decision making process by considering uncertainties of EV energy cost and also dynamics of itinerary information. Detailed main contributions of this paper are divided into four parts and are summarized as follows:

- A multi-channel stochastic energy cost prediction framework: This framework is learned and constructed by combining the essential vehicle physical model and the analysis of numerous real-world collected data.

- A real-time updating algorithm for the energy cost prediction model: This algorithm is proposed to extend the online learning capability of the prediction model for electric vehicles in real-world applications and conditions.

- Multistage charging decision-making models: Both of the deterministic and robust models are constructed for average outcome decision-making and safe charging strategies, respectively. Here the term "safe" means that proposed charging strategies can always make sure CAEVs have enough energy to finish a desired itinerary with consideration of uncertainties of energy cost in realistic situations.

- A dynamic programming algorithm for optimal charging strategies: This algorithm helps CAEVs to obtain the charging strategies dynamically at each visited location with a lower computational cost.

\section{Data-Driven Multi-Channel Stochastic Energy Cost Prediction}

\subsection{The EV Project Data Set}

Idaho National Laboratory partnered with ECOtality, Nissan, General Motors, and more than 10,000 other city, regional and state governments, electric utilities, other organizations and members of the general public, to deploy over 
12,000 AC Level 2 (208-240V) charging units and over 100 dual-port DC fast chargers in 20 metropolitan areas. Approximately 8,300 Nissan LEAF, Chevrolet Volts, and Smart ForTwo Electric Drive vehicles were also enrolled in the project. The EV Project is a large deployment and evaluation project of electric drive vehicles and charging infrastructure in United States. Project participants gave written consent for EV Project researchers to collect and analyze data from their vehicles and/or charging units. The data collection phase of The EV Project ran from January 1, 2011, through December 31, 2013 and captured almost 125 million miles of driving and 4 million charging events. Idaho National Laboratory is responsible for analyzing the data collected and publishing results. The detailed information of this project and data set can be found in INL (2013) and Smart and Schey (2012). In the subsequent analyses and modeling, only the dataset of Nissan Leaf are utilized.

\subsection{EV Energy Consumption Analysis and Data Demonstration}

As we introduced in the the literature review, many previous research has been performed to estimate the energy cost. One of the important methods utilizes the vehicle longitudinal dynamic model. The longitudinal tractive force can be modeled as:

$$
F(v)=\frac{1}{2} \rho C_{d} A(v-w)^{2}+C_{r} M g v \cos (\alpha)+\tilde{M} a+M g \sin (\alpha)
$$

In this equation, $v$ is vehicle speed, $C_{d}$ denotes the air drag coefficient, $A$ is the projected frontal area, $w$ is the wind speed component in the direction of vehicle speed, $\rho$ is the air density, $M$ is the vehicle mass, $C_{r}$ is the coefficient of rolling resistance, $\alpha$ is the angle of road surface for the slope or grade, $\tilde{M}$ is the equivalent mass considering the inertial mass factors and $a$ is the vehicle acceleration. The detailed analysis of this equation can be found in Yi and Bauer (2017b).

The longitudinal dynamical model describes tractive force coupled with a speed profile. It describes wheel energy or power consumption depending on several realistic conditions, for example, traffic condition, environmental condition, terrain. From the real world data demonstration in Figure 1, we can see that there exist huge uncertainties for the energy cost for the same distance of trip. In fact, it is very difficult to obtain all these real time data to estimate an accurate energy cost along a road segment. In real-word operation, an approximate stochastic model that can provide the average energy cost and the corresponding uncertainties may be good enough for charging decision making. Once we can know the uncertainty pattern of energy cost, we can provide the optimal strategies for overall average outcome or safe charging strategies for worst case considerations. Uncertainties of EV energy cost is especially important when EVs have low state of charge. Most of charging actions may happen when EVs have battery low state of charge. These uncertainties may have big effect on the final battery energy state after some trips and also the future potential energy demand for an itinerary. In order to provide more accurate and 


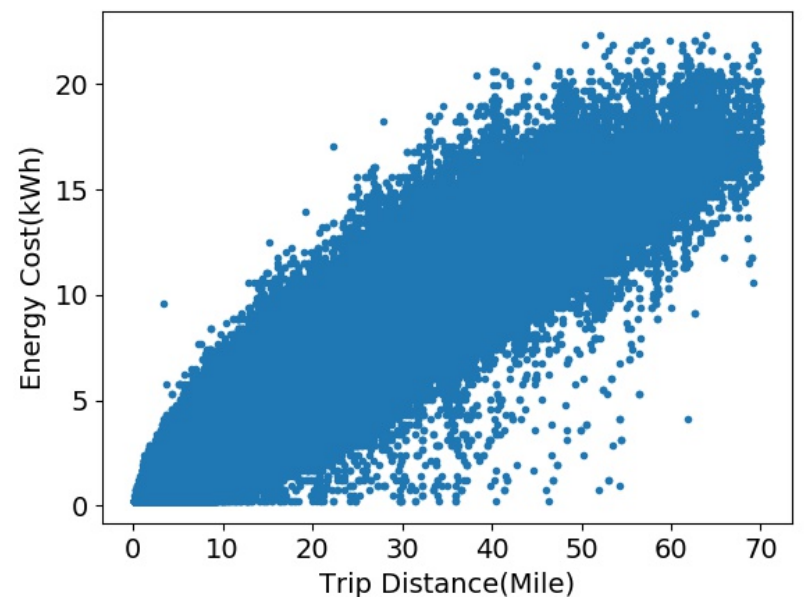

Figure 1: Trip energy cost versus distance for Nissan Leaf in EV Project

globally optimal charging strategies, it is important and necessary to consider and investigate these uncertainties in the energy consumption prediction model.

From the longitudinal dynamic model, the energy cost per mile should have an approximate quadratic relationship with vehicle speed under constant vehicle speed, as shown in Equations (2) and (3), where $E_{c}$ is the energy cost during a trip segment, $E_{a c}$ is the energy cost of accessory loads, $d$ is the distance of trip segment, $\eta$ is the powertrain efficiency and $\gamma_{0}, \gamma_{1}, \gamma_{2}$ are the coefficients obtained from Equation (1). This function can model the average energy cost of EV along a road segment.

$$
\begin{gathered}
E_{c}=\eta(F(v) d)+E_{a c} \\
E_{c} / d=\eta\left(\gamma_{2} V^{2}+\gamma_{1} V+\gamma_{0}\right)+\frac{E_{a c}}{d}
\end{gathered}
$$

Figure 2 demonstrates the distribution for energy cost per mile with regard to different vehicle speeds. Box plot is utilized to illustrate the uncertainties under different average speeds. In this figure, the entire average speed range is divided into 20 intervals equally. We can see that the average cost has an approximate quadratic relationship with average vehicle speed. But the uncertainties or variances are very different under different average speeds and accessory loads. Lower vehicle speeds have larger uncertainties. It is reasonable that city driving situations with low speeds can be affected more by traffic and environmental conditions and accessory loads. In order to characterize more details of this model and describe the uncertainties in a more accurate way, it is necessary to provide individual uncertainty models for different average speeds. 


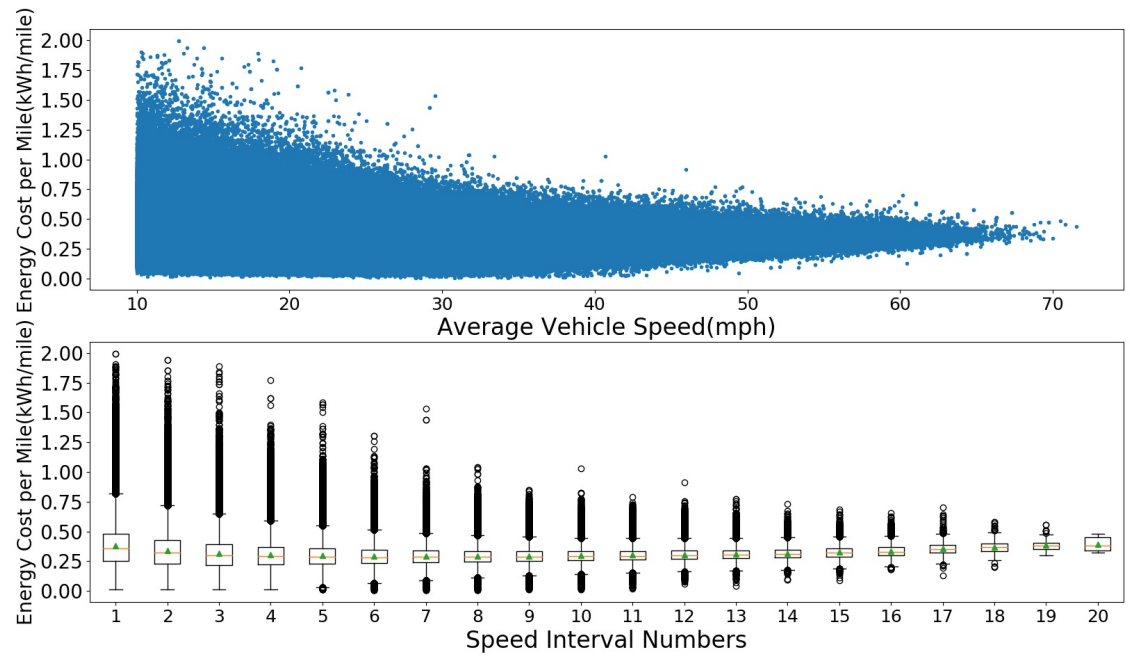

Figure 2: Energy cost per mile with regard to average speed and the corresponding box plot with regard to 20 speed intervals

\subsection{Multi-Channel Stochastic Energy Consumption Model}

As shown in Figure 2, with various average speeds on trip segments, uncertainties of energy cost have much difference due to several realistic situations, e.g. traffic and environmental conditions. We need to construct various energy consumption models for different driving speed conditions. The whole range of vehicle speed is divided into several segments. Assume that the energy cost model for each speed interval follows the same random pattern. We can have the corresponding values of mean and variance of stochastic model for each speed interval. More intervals can provide high-resolution energy consumption prediction model. The following is a discretization with $N$ speed intervals.

$$
\mathcal{V}=\left[v_{0}, v_{1}\right] \bigcup\left(v_{1}, v_{2}\right] \bigcup \ldots \bigcup\left(v_{N-1}, v_{N}\right]
$$

where $v_{0}$ is the minimum speed value $v_{\min }$ and $v_{N}$ is the maximum speed value $v_{\max }$. Suppose each sub interval $\mathcal{V}_{i}=\left(v_{i-1}, v_{i}\right]$ has the corresponding mean $m_{i}$ and variance $\sigma_{i}^{2}$ for energy cost per mile on each trip segment. Then we can have the mean value set for energy cost per mile $\mathbf{m}=\left\{m_{1}, m_{2}, \ldots, m_{N}\right\}$ and variance value set for energy cost per mile $\sigma^{2}=\left\{\sigma_{1}^{2}, \sigma_{2}^{2}, \ldots, \sigma_{N}^{2}\right\}$. In order to obtain the mean and variance set for energy cost per mile, the entire data set has been divided into $N$ subsets according to the average speed. In each subset, the mean and variance of energy cost per mile is calculated. They are illustrated in Figure 3 and Figure 4. From data points of mean and variance, we can fit them and derive the prediction function for mean value and variance 


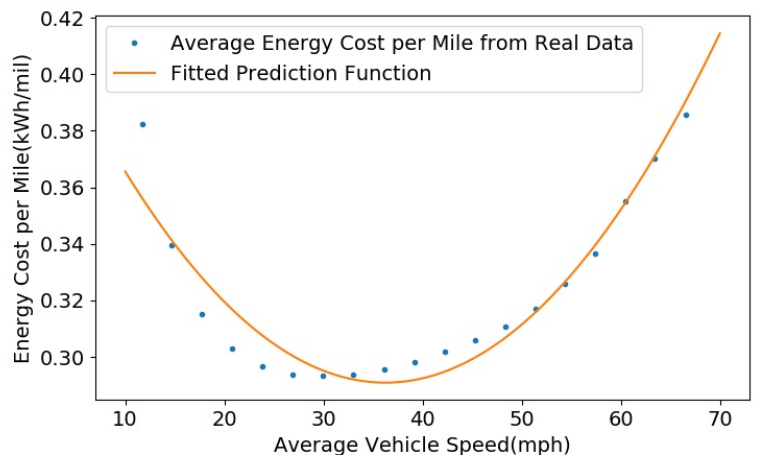

Figure 3: Average energy cost per mile prediction function

on any specific vehicle speed in the given range. The specific fitted functions obtained from EV project data set are as follows:

- Mean prediction function of energy cost with regard to vehicle speed

$$
F_{m p}(v)=0.0001085 v^{2}-0.007861 v+0.4334
$$

- Variance prediction function of energy cost with regard to vehicle speed

$$
F_{v p}(v)=0.09072504 e^{-0.097365332 v}+0.00218884
$$

We need to clarify that these models from EV project data set are not universal. This is just a demonstration for Nissan Leaf from the data we have. But the modeling process is applicable for other electric vehicles. The fitted functions are shown in Figure 3 and Figure 4. Mean value prediction function is a quadratic function and the variance prediction function is a exponential-like function. By using these two functions, mean value and variance for any vehicle speed in the given range will be derived.

Energy consumption prediction in illustrated models only utilizes the vehicle speed as the input. The reason that only one factor used is due to the resolution limitation of the EV Project Dataset. Energy consumption of EVs depends on several other realistic conditions, for example, temperature, vehicles load and powertrain efficiency, etc. However, the proposed data-driven methodology in this paper has the capability to consider other relevant features if the corresponding data is collected. Generally it can be a multi-dimensional and multichannel stochastic model according to the available real world data. Currently the available data can only help us to create a one-dimensional multi-channel stochastic model with regard to vehicle speed. In the future, we will try to collect more detailed energy consumption data under different realistic conditions and construct multi-dimensional multi-channel stochastic model, for example, two-dimensional multi-channel model with consideration of both vehicle speed 


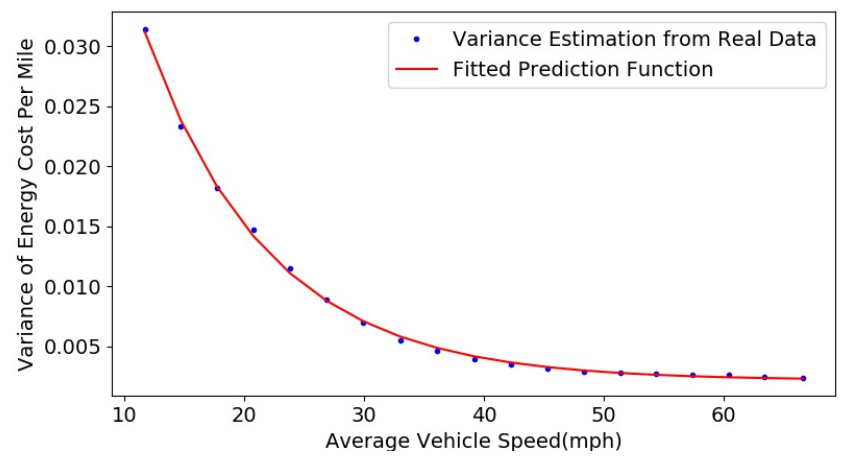

Figure 4: Variance prediction function for energy cost per mile with regard to vehicle speed

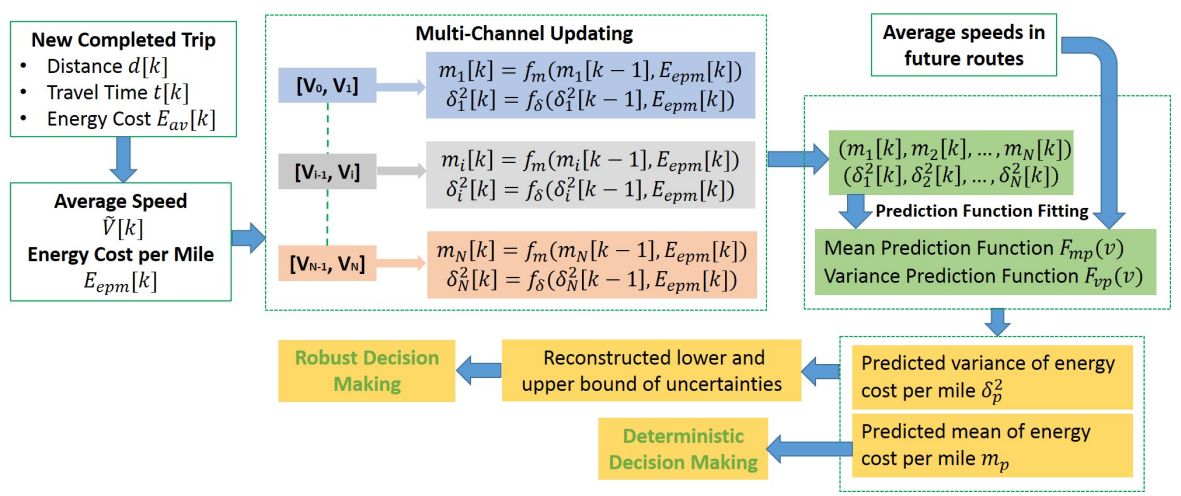

Figure 5: Overall framework for multi-channel energy consumption prediction model

and ambient temperature condition, or three-dimensional multi-channel model with consideration of speed, vehicles load and ambient temperature, etc. Therefore, the introduced framework definitely has the capability to construct more accurate energy consumption models.

\subsection{Real-Time Updating Algorithm for Energy Consumption Prediction}

In previous analyses, we have utilized the historical data set to construct the stochastic energy consumption model. But in realistic situations, we cannot have the detailed dataset for each EV at the beginning. But data can be collected all the time for each vehicle when the vehicles are being driven and realistically, a base model would be in-place from an OEM. Therefore, a real-time updating algorithm for energy consumption prediction model is necessary for optimization in real-world applications. In this paper, the real-time updating framework in Figure 5 is proposed and evaluated.

For each new completed trip, we can have the distance $d[k]$, the travel time $t[k]$ and the corresponding energy cost $E_{a v}[k]$. Average speed $v[k]$ and energy 

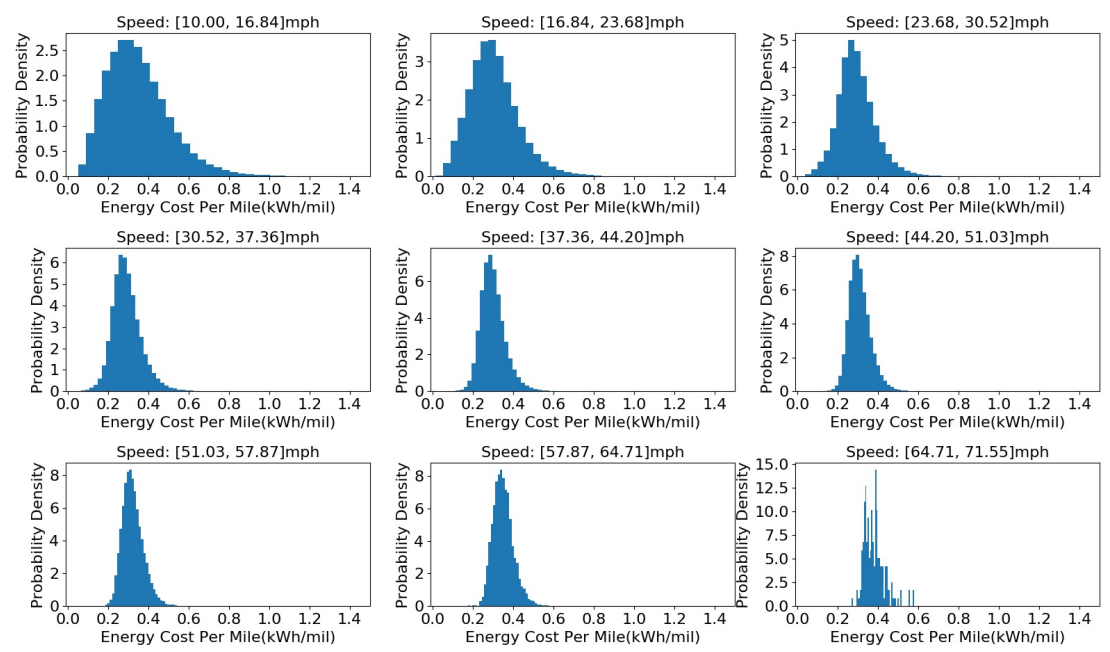

Figure 6: Distributions of energy cost per mile in nine average vehicle speed intervals

cost per mile $E_{\text {epm }}[k]$ on this trip segment are derived. According to the average speed, the channel $i$ to be updated is determined at time step $k$. Denote the mean value and variance of channel $i$ at step $k$ are $m_{i}[k]$ and $\sigma_{i}^{2}[k]$, respectively. The following equations are used to update the mean value $m_{i}[k]$ and variance $\sigma_{i}^{2}[k]$ in an online manner. The detailed functional analysis and proof for this algorithm can be found in Welford (1962).

$$
\begin{gathered}
m_{i}[k]=\frac{k-1}{k} m_{i}[k-1]+\frac{1}{k} E_{\text {epm }}[k] \\
\sigma_{i}^{2}[k]=\frac{k-1}{k} \sigma_{i}^{2}[k-1]+\frac{k-1}{k^{2}}\left(E_{\text {epm }}[k]-m_{i}[k-1]\right)^{2}
\end{gathered}
$$

With the power of this algorithm, the mean values and variance can be calculated iteratively in a real-time way. At each step $k$, we can utilize the obtained estimation to find the up-to-date prediction functions, which provide the corresponding predictions $m_{p}$ and $\sigma_{p}^{2}$.

Once mean value and variance of energy cost per mile are predicted for a given vehicle speed, we can use them to construct the corresponding lower bound as $E_{l w}$ and upper bound as $E_{u p}$ for the description of uncertainties. Figure 6 illustrates the distributions of nine average vehicle speed intervals. Since the energy cost per mile is always positive, based on the histogram results in Figure 6, the log-normal distribution is a reasonable assumption to describe the uncertainties. According to these log-normal distributions, we can calculate the lower bound and upper bound for each average speed. For example, if we have the mean value of $m_{p}$ and variable $\sigma_{p}^{2}$, denote the corresponding distribution 


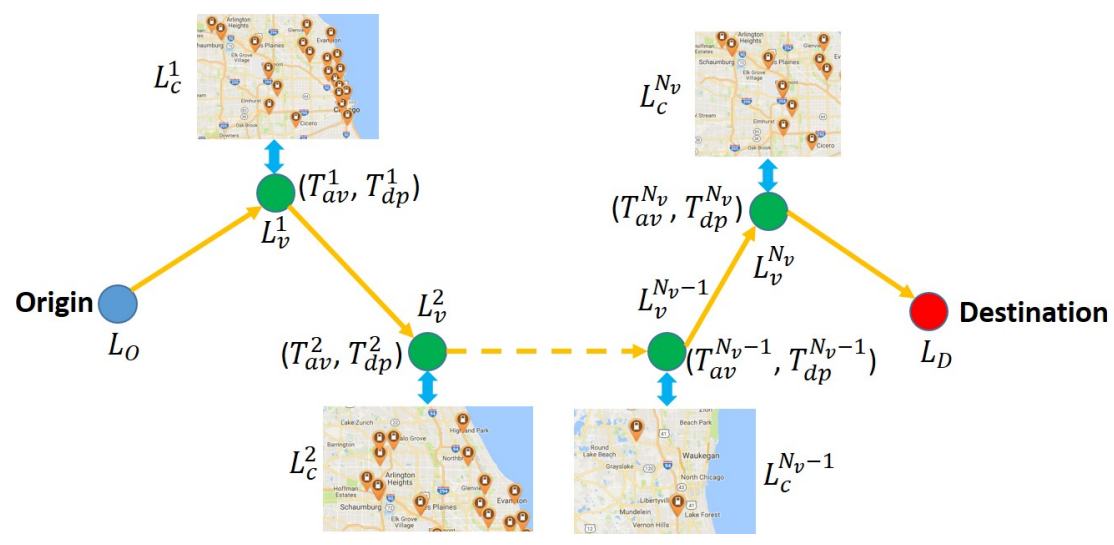

Figure 7: A Model for daily itinerary

of energy cost per mile as $E_{p m} \sim \operatorname{Lognormal}\left(m_{p}, \sigma_{p}^{2}\right)$ is obtained. Suppose the cumulative distribution function is $P_{r}\left(E_{p m}\right)$, we can define the lower bound $E_{l w}$ as $P_{r}\left(E_{p m} \leq E_{l w}\right) \leq 0.05$ and upper bound $E_{u p}$ as $P_{r}\left(E_{p m} \geq E_{u p}\right) \geq 0.95$. Here the probability interval $[0.05,0.95]$ is just an example, it can be defined according to realistic situations. This is similar to the confidence interval.

The lower bound and upper bound of energy cost per mile aid in determining prediction uncertainties for each future trip segment. By considering these uncertainties, the following proposed robust decision-making model will design a safe charging strategy in case of some random high energy costs along the route. Only the average speed information is utilized to illustrate of the uncertainty of energy cost prediction model due to the resolution limit of EV project data set. More detailed real-world data set could tighter the uncertainty if known a priori, i.e. temperature. This means that the proposed method is extensible to obtain more accurate energy consumption prediction model with multiple input variables.

\section{Charging Decision-Making Optimization Models}

\subsection{Modeling}

\subsubsection{Connected and Automated Electric Vehicle}

This paper considers the charging decision making for connected automated electric vehicles. Here the "connected" and "automated" means at least two aspects: (1) electric vehicles are connected with the infrastructure network and have the information from nearby charging stations, for example, location, charging level, waiting time, price, etc. (2) Electric vehicles are fully autonomous vehicles and can perform the charging actions by themselves. Based on these assumptions, electric vehicles can have totally different charging behaviors because drivers are not required to be present at the charging station. 


\subsubsection{Itinerary Modeling}

A typical personal daily itinerary starts from home and ends at home. In order to consider different situations and make sure the extensibility of proposed models, an investigated itinerary considered in the optimal charging decision making framework, as shown in Figure 7 is assumed to have the following information:

- Origin location $L_{O}$ and destination location $L_{D}$ : Different origin and destination information represent different scenarios. The most common one for a daily itinerary is that both of origin and destination are home.

- Intermediate visited location set $\mathcal{L}_{v}$ and $\left|\mathcal{L}_{v}\right|=N_{v}$. At each visiting location $L_{v}^{k}$ and $1 \leq k \leq N_{v}$, vehicle owner can have the corresponding arrival time $T_{a v}^{k}$ and departure time $T_{d p}^{k}$. Then the available time for charging action is $T_{u c}[k] \leq T_{d p}^{k}-T_{a v}^{k}$.

- Initial energy state at origin and the requirement of energy state at destination: Assume the initial energy state is known at the origin. The energy state requirement of destination will influence the charging strategies along the itinerary. If the destination is at home, we can assume that no specific energy state value is required for electric vehicles due to the capability of charging at home.

\subsubsection{Charging Infrastructure Network}

Due to the assumption of connected vehicles, CAEVs can obtain the following information from charging infrastructure network:

- Location information of nearby charging stations. The location set of charging stations near $L_{v}^{k}$ is denoted as $\mathcal{L}_{c}^{k}$ and $\left|\mathcal{L}_{c}^{k}\right|=N_{c}^{k}$. Here we assume that CAEVs only perform the charging actions in charging stations which are in a certain driving range from $L_{v}^{k}$.

- Each charging station $i, 0 \leq i \leq N_{c}^{k}$ provides the information of charging level or maximum charging power $P_{c}^{i}$, charging price $P_{r}(t)$, waiting time $T_{w}(t)$ and the corresponding charging function. There are different charging price models. In this paper, we utilize the price model as dollar $/ k W h$ in the decision-making framework. It can easily be extended to other price models.

\subsection{Optimal Decision-Making Formulation}

The charging decision-making needs to provide charging strategies based on the battery energy state and travel demand for an investigated trip. Charging strategies include the amount of charged energy and also the selected charging station near each visited location. The mathematical description for this problem will be given as follows. A scheme in Figure 8 illustrates the connections between these mathematical model parts. 


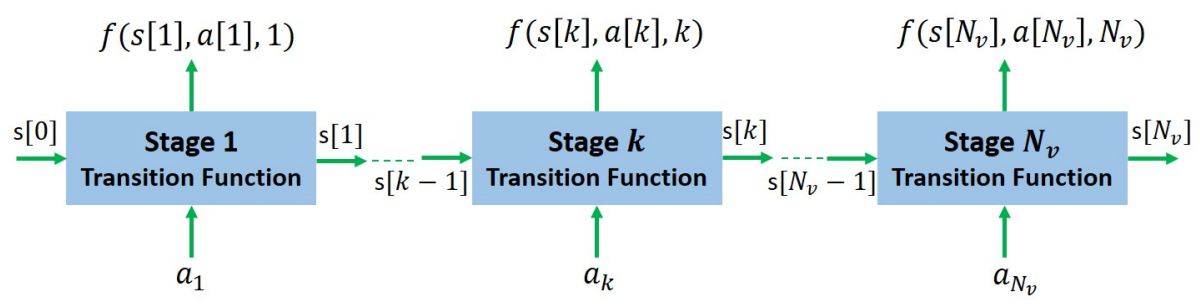

Figure 8: A scheme of dynamic decision making process

$N_{v}$ visited locations except home are investigated in the given itinerary. Define there are in total $N_{v}$ stages for the entire decision-making process. Denote a specific stage as $k$, where $1 \leq k \leq N_{v}$.

(1) State $s[k]$ :

$s[k]$ is the battery energy state of CAEV when arrived at a visited location $L_{v}^{k}$ during a daily itinerary. This can be the state after charged for backward algorithm or the state before charged for forward algorithm in multistage decision-making.

(2) Decision action $a_{k}=\left\{E_{c}[k], \vec{x}[k]\right\}$ :

$E_{c}[k]$ is the amount of charged energy when EV user at location $L_{v}^{k} \cdot \vec{x}[k]$ has $N_{c}[k]$ elements. $N_{c}[k]$ is the number of available charging station in a neighborhood area. Each $x^{i}[k]$ in $\vec{x}[k]$ can only be 0 or 1 . And $\sum_{i=1}^{N_{c}} x^{i}[k] \leq 1$. This means the vehicle can only utilize at most one charging station to obtain the required energy. Of course, when the available charging time is long enough, maybe CAEV can complete the charging actions at different charging stations. Therefore, we have $E_{c}[k]=\sum_{i=1}^{N_{c}^{i}} E_{c}^{i}[k] x^{i}[k]$, where $E_{c}^{i}[k]$ is the charged energy in charging station $i$ at stage $k$.

(3) Utility function at stage $k$ :

$$
f(s[k], a[k], k)=\sum_{i=1}^{N_{c}[k]} E_{c}^{i}[k] P_{r}^{i}[k] x^{i}[k]+\lambda \sum_{i=1}^{N_{c}[k]} E_{c c}^{i}[k] x^{i}[k]
$$

This utility function aims to calculate the monetary cost of energy charged away from home and also the additional travel energy cost when performing charging actions. Minimizing this utility function aims to improve the sustainability of both monetary and energy costs. In this function, besides the variables we introduce in previous parts, $E_{c c}^{i}[k]$ is the transportation energy cost for the charging action to the nearby charging station $i . P_{r}^{i}[k]$ is the charging price by dollar $/ k W h$ in charging station $i . \lambda$ is a weighting factor.

(4) Transition functions:

Denote $E_{t c}^{(k, k+1)}$ is the trip energy cost from location $L_{v}^{k}$ to location $L_{v}^{k+1}$,

- Forward state transition (used for final state fixed)

$$
s[k+1]=s[k]+E_{c}[k]-E_{t c}^{(k, k+1)}-2 \sum_{i=1}^{N_{c}^{k}} E_{c c}^{i}[k] x^{i}[k]
$$


where $s[k]$ is the battery energy state before charging action performed, $E_{c}[k]$ is the energy charged at stage $k, E_{c c}^{i}[k]$ is the energy cost for performing charging action from location $L_{v}^{k}$ to charging station $L_{c}^{i}[k]$.

- Backward state transition (used for initial state fixed)

$$
s[k]=s[k+1]-E_{c}[k+1]+E_{t c}^{(k, k+1)}+2 \sum_{i=1}^{N_{c}^{k+1}} E_{c c}^{i}[k+1] x^{i}[k+1]
$$

where $s[k]$ is the battery energy state after charging action performed, $E_{c}[k+1]$ is the energy charged at stage $k+1, E_{c c}^{i}[k+1]$ is the energy cost for performing charging action from location $L_{v}^{k+1}$ to charging station $L_{c}^{i}[k+1]$.

(5) Overall utility function $U\left(s\left[N_{v}\right], a\left[N_{v}\right]\right)$ :

Overall cost for charging actions along the entire itinerary outside home is the summation of utility cost on each stage $k$.

$$
U\left(s\left[N_{v}\right], a\left[N_{v}\right]\right)=f(s[0], a[0], 0)+\sum_{k=1}^{N_{v}} f(s[k], a[k], k)
$$

where $f(s[0], a[0], 0)$ is the initial cost at home. In our case, $f(s[0], a[0], 0)=$ 0 without consideration of charging actions at home. It is included for the completeness of formulation.

\subsection{Optimization Models}

\subsubsection{Deterministic Case}

In the deterministic case, we utilize an average energy cost for each trip segment. The uncertainties of energy cost on each trip segment are not considered in this optimization model. This kind of model will provide the decision-making strategies in an average sense. The optimization model is constructed as follows:

$$
\begin{array}{ll}
\min _{a[1], \ldots, a\left[N_{v}\right]} & f(s[0], a[0], 0)+\sum_{k=1}^{N_{v}} f(s[k], a[k], k) \\
\text { s.t. } & s[0]=S_{0} \\
& s[k-1]=s[k]-\sum_{i=1}^{N_{c}^{k}} E_{c}^{i}[k] x^{i}[k]+E_{t c}^{(k-1, k)}+2 \sum_{i=1}^{N_{c}^{k}} E_{c c}^{i}[k] x^{i}[k] \\
& P_{d}[k] \leq s[k] \leq C_{p} \\
& 0 \leq E_{c}^{i}[k] \leq E_{u p r}^{i}[k] \\
& x^{i}[k]=0 \text { or } 1, \quad \text { and } \sum_{i=1}^{N_{c}^{k}} x^{i}[k] \leq 1 \\
& k=1, \ldots, N_{v}
\end{array}
$$


Besides the formulations included in previous sections, some more constraints are introduced in this optimization model:

(1) $P_{d}[k] \leq s[k] \leq C_{p}$ is the constraint of battery energy state. $C_{p}$ is the battery capacity and $P_{d}[k]$ is the energy demand prediction for future trips at stage $k$. For example:

- One-step prediction

$$
P_{d}[k]=E_{t c}^{(k, k+1)}
$$

- Two-step prediction

$$
P_{d}[k]=E_{t c}^{(k \rightarrow k+1)}+E_{t c}^{(k+1, k+2)}
$$

(2) $0 \leq E_{c}^{i}[k] \leq E_{u p r}^{i}[k]$ is the constraint for charged energy. $E_{u p r}^{i}[k]$ is the available maximum energy obtained from charging station $i$ at stage $k$.

$$
E_{u p r}^{i}[k]=f_{c r}^{i}\left(s[k-1]-E_{t c}^{(k-1, k)}-E_{c c}^{i}[k], T_{c}^{i}[k]\right)
$$

This function is used to determine the maximum amount of available energy from charging station $i$. It depends on current battery energy state and also the available charging time $T_{c}^{i}$. This is because the charging rate may not be constant and it has a nonlinear relationship with regard to the battery energy state. The available charging time can be calculated by $T_{c}^{i}[k]=T_{u c}[k]-2 \frac{d^{i}[k]}{v^{i}[k]}-$ $T_{w}^{i}[k]$, where $T_{u c}[k]$ is the total time that CAEV user can spend at location $L_{v}^{k}, d^{i}[k]$ and $v^{i}[k]$ are the distance and average speed respectively to charging station $i$ from location $L_{v}^{k}$. In order to obtain the maximum available charge, we need to consider the charging rate function of charging station $i$. We can say charging stations of different levels can have different charging rate functions. In realistic situations, particularly for fast charging, it is not constant depending on battery state of charge. In the simulations of this paper, we approximate that charging power is constant. However, it can be extended to other non-constant situations, because the $E_{u p r}^{i}[k]$ is just an input of this decision-making model.

\subsubsection{Robust Case}

From the analysis for energy consumption model, the energy cost of each trip segment has uncertainties due to the traffic conditions, environmental and terrain information. In order to obtain the safe strategies for completing the itinerary with enough energy, these uncertainties are necessary to be involved in the decision-making model.

Equation (11) provides a robust optimization to consider the uncertainties in energy cost. This is a worst case optimization model. In this model, the trip energy costs have the lower bound and upper bound for both regular trips and the trips for charging actions, for example, $E_{t c}^{(k, k+1)} \in\left[E_{l w}^{k}, E_{u p}^{k}\right]$ and $E_{c c}^{i}[k] \in$ $\left[E_{l w}^{i}[k], E_{u p}^{i}[k]\right]$. These lower and upper bounds for uncertainties can be derived from the proposed bound reconstruction algorithm in Section 3. 


$$
\min _{a[1], \ldots, a\left[N_{v}\right]} \max _{\substack{E_{t c}^{(k, k+1)} \in\left[E_{l w}^{k}, E_{u p}^{k}\right] \\ E_{c c}^{i}[k] \in\left[E_{l w}^{i}[k], E_{u p}^{i}[k]\right]}} f(s[0], a[0], 0)+\sum_{k=1}^{N_{v}} f(s[k], a[k], k)
$$

s.t. Constraints in Equation (10)

Equation (11) has the same constraints as Equation (10). The considerations of uncertainties for energy cost makes this decision-making model have the capability to find safer charging strategies in real situation.

\section{Dynamic Programming for Optimal Strategies}

\subsection{Deterministic Case}

The objective for the optimization model in Equation (10) is to minimize the overall cost for charging actions outside home. The task is to find a sequence of actions, $a[1], \ldots, a\left[N_{v}\right]$ that minimize the overall utility function. Dynamic programming technology is promising to solve this type of multistage problem. Dynamic programming in this case involves the recursive formulation of the costto-come from an initial state. Assume we don't consider the charging action at home, we can say the initial cost at home is 0 . This mean that $a[0]=0$. Suppose that the initial cost is defined as $U(s[0], a[0])=0$.

The initial optimal cost-to-come is:

$$
U_{0,0}^{*}(s[0])=U(s[0], a[0])
$$

For an intermediate stage, $K \in\left\{1, \ldots, N_{v}\right\}$ the following represents the optimal cost-to-come:

$$
U_{0, K}^{*}(s[K])=\min _{a[0], \ldots, a[K]} U(s[0], a[0])+\sum_{k=1}^{K} f(s[k], a[k], k)
$$

According to the principle of optimality, we can have the following relationship

$$
U_{0, K}^{*}(s[K])=\min _{a^{*}[0], \ldots, a^{*}[k-1], a[K]} U_{0, K-1}^{*}(s[K-1])+f(s[K], a[K], K)
$$

Based on this equation, the following algorithm is proposed to solve this problem: 
Algorithm 1: Dynamic Programming for Multistage Charging Decision-
Making

(1) When $k=0$ :

$U(s[0], 0)=0$ and $a[0]=0$;

(2) When $k \geq 1$ :

Using this equation:

$$
U_{0, k}^{*}(s[k])=\min _{a^{*}[0], \ldots, a^{*}[k-1], a[k]} U_{0, k-1}^{*}(s[k-1])+f(s[k], a[k], k)
$$

Solve the following static problem for action $a^{*}[k]$ in stage $k$

$$
\begin{array}{ll}
\min _{a[k]} & U_{0, k-1}^{*}(s[k-1])+f(s[k], a[k], k) \\
\text { s.t. } & s[k-1]=s[k]-\sum_{i=1}^{N_{c}^{k}} E_{c}^{i}[k] x^{i}[k]+E_{t c}^{(k-1, k)}+2 \sum_{i=1}^{N_{c}^{k}} E_{c c}^{i}[k] x^{i}[k] \\
& P_{d}[k] \leq s[k] \leq C_{p} \\
& 0 \leq E_{c}^{i}[k] \leq E_{u p r}^{i}[k] \\
& x^{i}[k]=0 \text { or } 1, \text { and } \sum_{i=1}^{N_{c}^{k}} x^{i}[k] \leq 1
\end{array}
$$

(3) Let $k=k+1$, if $k \leq N_{v}$, return step (2).

\subsection{Robust Case}

Similar to the deterministic case, the following relationship for a robust decision-making model can be derived according to the principle of optimality.

$$
U_{0, K}^{*}(s[K])=\min _{a[K]} \max _{\substack{E_{t c}^{(k, k+1)} \in\left[E_{l w}^{k}, E_{u p}^{k}\right] \\ E_{c c}^{i}[k] \in\left[E_{l w}^{i}[k], E_{u p}^{i}[k]\right]}} U_{0, K-1}^{*}(s[K-1])+f(s[K], a[K], K)
$$

According to this relationship, we can utilize the similar procedure in Algorithm 1 to find the optimal charging strategies. The above equation is a static optimization problem. In order to solve this optimization problem, we need to perform an equivalent transformation. It has the following equivalent optimization model: 


$$
\begin{array}{ll}
\min _{a[k]} & v \\
\text { s.t. } \quad & s[k-1]=s[k]-\sum_{i=1}^{N_{c}^{k}} E_{c}^{i}[k] x^{i}[k]+E_{t c}^{(k-1, k)}+2 \sum_{i=1}^{N_{c}^{k}} E_{c c}^{i}[k] x^{i}[k] \\
& P_{d}[k] \leq s[k] \leq C_{p} \\
& 0 \leq E_{c}^{i}[k] \leq E_{u p r}^{i}[k] \\
& U_{0, k-1}^{*}(s[k-1])+f(s[k], a[k], k) \leq v \\
& E_{l w}^{i}[k] \leq E_{c c}^{i}[k] \leq E_{u p}^{i}[k] \\
& E_{l w}^{k} \leq E_{t c}^{(k, k+1)} \leq E_{u p}^{k} \\
& x^{i}[k]=0 \text { or } 1, \quad \text { and } \sum_{i=1}^{N_{c}^{k}} x^{i}[k] \leq 1, \quad i=1, \ldots, N_{c}^{k}
\end{array}
$$

During each stage $k$, we need to solve the Equation (13) to obtain the robust optimal charging action $a[k]$. In this equivalent model, energy cost components are considered to be decision variables with lower and upper bounds. This model has involved the uncertainties into charging decision-making.

The equivalent transformation from Equation (11) (min-max optimization problem) to Equation (13) is a very common technology to solve the nonprobabilistic robust optimization model in Equation (11). The detailed explanation of this method can be found in Ben-Tal et al. (2009). This means that we can transfer a worst-case min-max optimization problem to a new general solvable optimization problem. Therefore, we have the new objective function and constraints as shown in Equation (13). This robust model tries to find the strategies for the worst case analysis. So this is a worst case decision making for safe charging decision strategies. This decision making model only takes use of the derived lower and upper bound of EV energy cost at each trip segment. Energy state of EV transits with some uncertainties (maybe some probability distributions), but the proposed model only considers boundary values of energy consumption to make sure that charging strategies must work even with the worst case of energy cost.

\section{Simulations and Case Study}

\subsection{Simulations for Real-Time Updating Framework}

In Section 3, we have proposed a multi-channel stochastic energy consumption prediction model to estimate the average energy cost and also the corresponding uncertainties. A real-time updating algorithm has been introduced for online model construction by using the new measured data points in future real-world applications. We have provided the data analysis description of the entire data set in Section 3. In this simulation, we would like to utilize the same data set to provide the evaluations of the real-time model updating algorithm. 


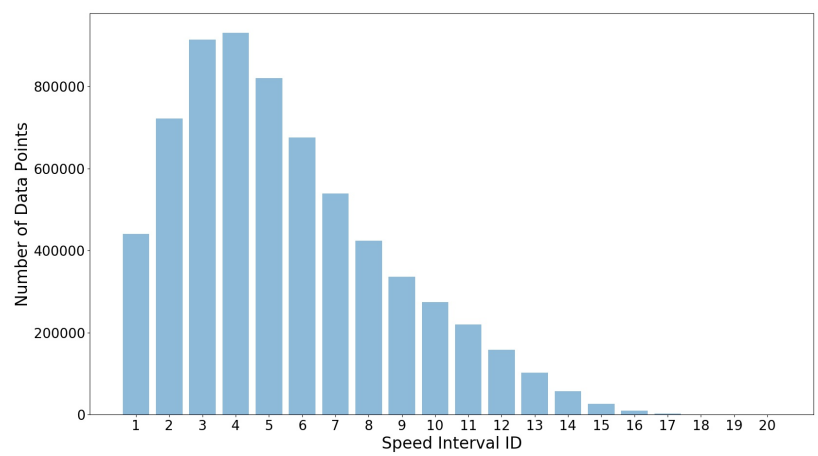

Figure 9: Number distribution of data points in 20 speed intervals

Table 1: Specific Numbers of Data Points for Selected Five Intervals

\begin{tabular}{ccc}
\hline Interval ID & Speed Interval $(\mathrm{mph})$ & Number of Data Points \\
\hline \hline 1 & {$[10.000,13.078]$} & 441345 \\
\hline 5 & {$[22.310,25.388]$} & 820688 \\
\hline 10 & {$[37.699,40.766]$} & 275171 \\
\hline 15 & {$[53.087,56.164]$} & 26137 \\
\hline 20 & {$[68.475,71.552]$} & 19 \\
\hline
\end{tabular}

Data samples from the EV project will be fed into the model one by one in a consecutive way. Each sample is a trip segment with an average speed. The entire vehicle speed range is divided into 20 sub intervals equally in this simulation. According to the rules proposed in the multi-channel framework, we can have the sample number distribution in each vehicle speed interval, as shown in Fig. 9. We can see that most of the vehicles in the EV Project are utilized in city driving situations. Table 6.1 provides the specific numbers of data points in five speed intervals that are utilized for demonstrations in the following results.

Fig. 10 illustrates the estimation traces of mean values of energy cost per mile for five different speed intervals. When more samples are utilized to update the estimation, mean values become stable. However, the traces have different patterns. This is because they have different number distributions in this EV project data set. Most of them come from city drive situations. In the intervals with relative low speed values, estimation values converge much faster due to much more data samples. The special case is the interval No.20 with the highest speed value in this data set. Only 19 samples exist. This is extremely small comparing to the entire data set with the size of more than 6 million samples. This introduces large fluctuations in the estimation process. Fig. 11 provides the estimations traces of variance of energy cost per mile for five different speed intervals, which are the same as Fig. 10. All these variances converge to the final values that are estimated by one time from the entire data set. The converge 


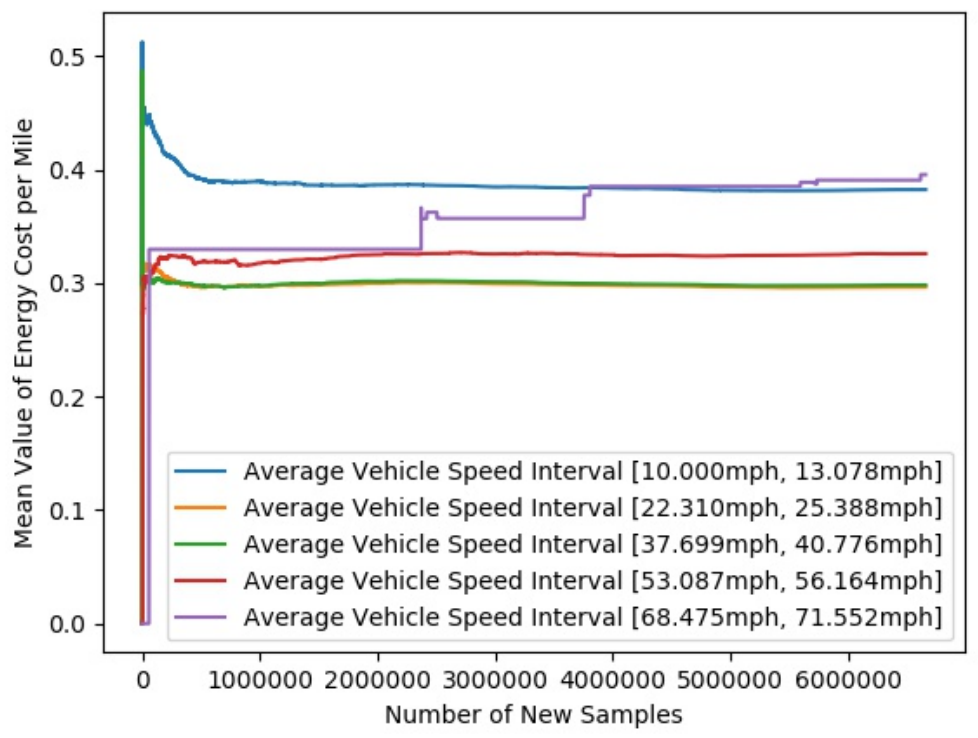

Figure 10: Mean value trace of energy cost per mile for different average speed intervals

rates have the similar patterns as Fig. 10. This is also due to the sample number distribution among different speed intervals. Both of these results demonstrate the capability of convergence for the proposed algorithm to the final stable values. It will be very useful to obtain the estimations in a real-time and low computational cost way.

Fig. 12 provides the function fitting results for average energy cost per mile with regard to different average speeds of trip segments. The results show that the changes are pretty small at low speeds and much difference can occur at high speed values when increasing the used data points. This is because most of data points have low average speed values and can converge to the stable values at the very beginning. The intervals with high average speeds need more data points to adjust and achieve the final state values. Fig. 13 illustrates the function fitting results for variance of energy cost per mile with regard to different average trip speed. Results for variance estimation show an opposite pattern to the mean value estimation. More differences occur at low speed values. Actually this is also caused by the number of data points. More data points can help to narrow down the uncertainties and then reduce the values of variance.

All simulation results in this section show the good capability to estimate the mean value and the corresponding variance in a real-time and adaptive manner. During each real-time step, we can utilize the obtained estimations to construct the up-to-date stochastic energy cost prediction model. This kind of adaptive method has the strong robustness to adapt new measured data points. 


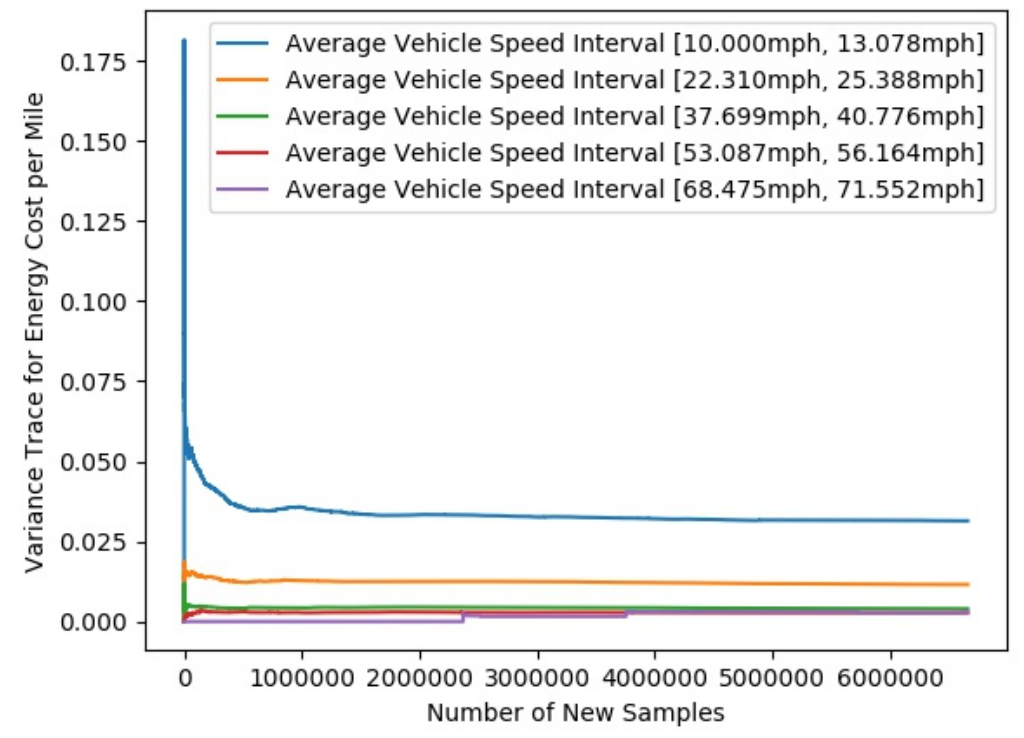

Figure 11: Variance value trace of energy cost per mile for different average speed intervals

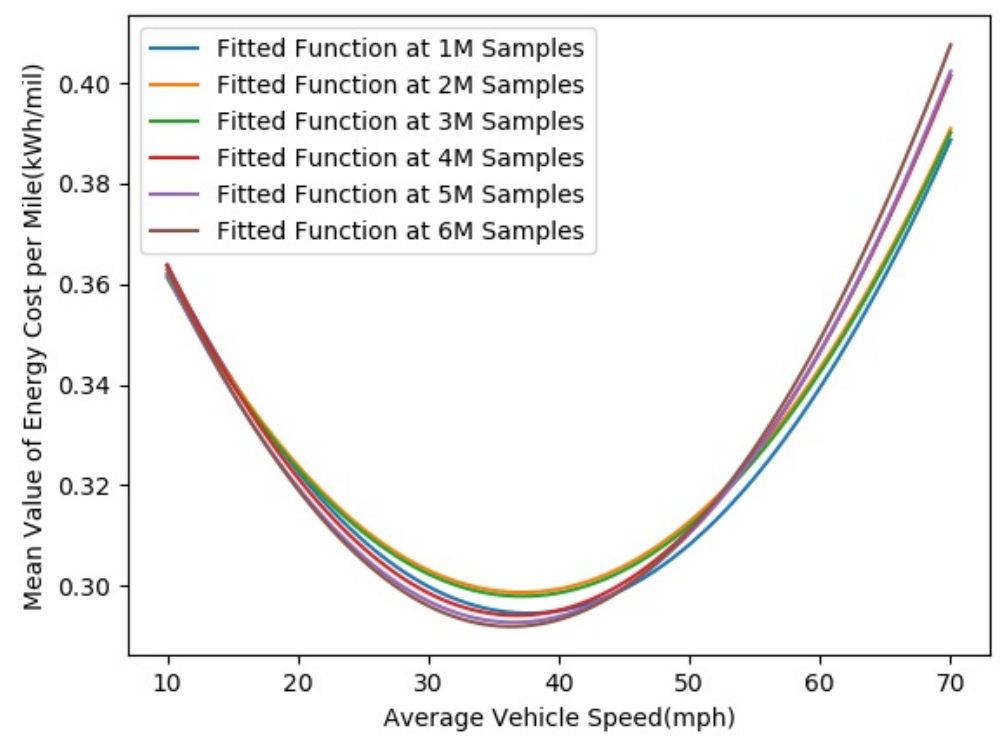

Figure 12: Mean value trace of energy cost per mile for different average speed intervals 


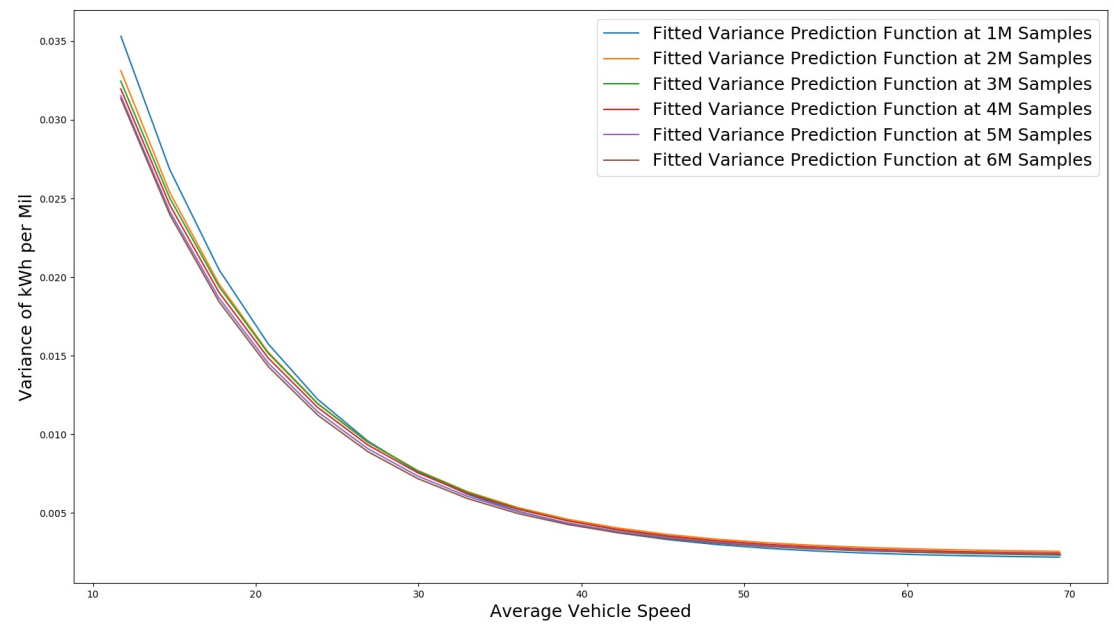

Figure 13: Variance value trace of energy cost per mile for different average speed intervals

This makes sure that current models can always describe the newest energy cost pattern and provide more accurate predictions.

\subsection{Case Studies for Optimal Charging Decision Making}

Case studies in this section are trying to demonstrate the functionality of proposed optimal decision-making model. Itineraries from a real-world data set "Chicago Regional Household Travel Inventory (CRHTI)" by CMAP (2007) in Chicago are utilized. It is a comprehensive study of the demographic and travel behavior characteristics of residents in the greater Chicago area. Two different case studies are illustrated in this section: First, demonstration of charging decision making strategies for a single selected itinerary; Second, charging analysis for the whole itinerary dataset of CRHTI.

\subsubsection{Case Studies for A Single Itinerary}

A daily itinerary is selected from "Chicago Regional Household Travel Inventory (CRHTI)". The selected itinerary is a related long distance itinerary during a weekday in Figure 14. This itinerary comes from a financial planner. It has an overall distance of about 172 miles. This type of long-distance itinerary can help to illustrate the power of our algorithm. It will show how the fully automated short-range EV could finish a long-distance daily itinerary by using the current public charging station network. In this daily itinerary, location 1 is the home, and there are six different visited locations during this day. Figure 14 provides the specific locations on Google Maps and also the corresponding arrival time and departure time. 


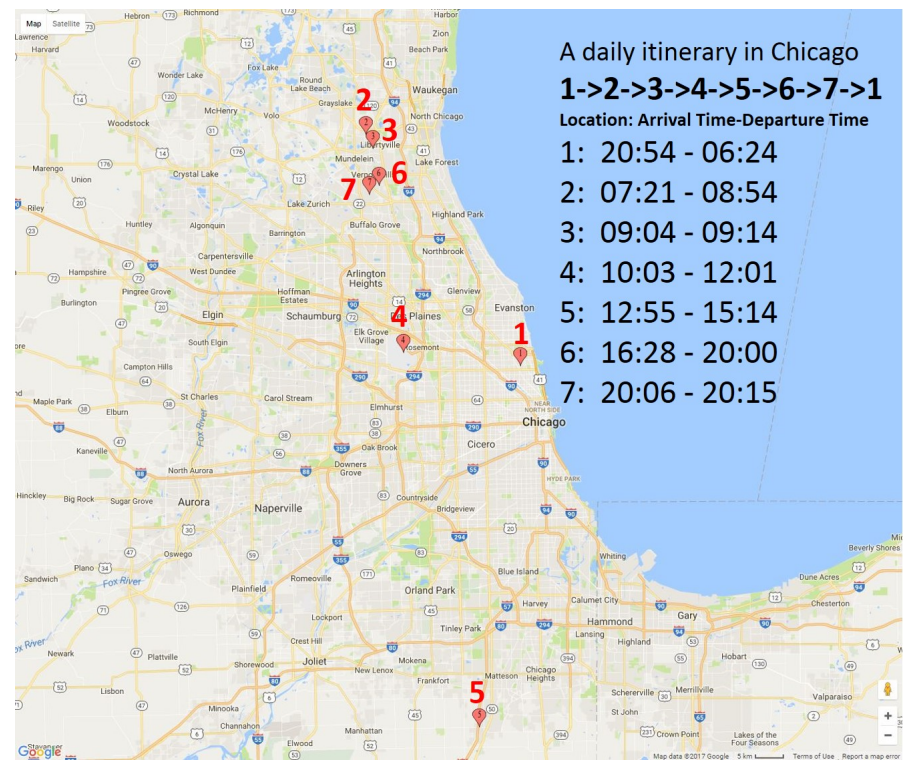

Figure 14: A daily itinerary in Chicago Regional Household Travel Inventory

Figure 15 and Figure 16 provide the location information of level 2 charging stations and DC fast charging stations respectively. Each visited location has its own neighborhood charging infrastructure network. The range constraint (range between charging stations and itinerary locations) used in this case study is 6 mile(about $10 \mathrm{~km}$ ). The range constraint of 6 miles is just an example. This constraint is determined by several realistic situations, for example, desirable maximum percentage of "empty vehicle miles traveled", density of charging stations, etc. In these figures, we have provided the specific information of latitude and longitude of six visited locations. The nearby charging station information is obtained from the website of Alternative Fuels Data Center from U.S. Department of Energy (AFDC (2017)). From these information, we can see that there are no charging stations at the visited locations. If this financial planner cannot leave this location to do the charging actions (this may always happen due to the business requirement), most short-range EVs, e.g. Nissan Leaf, cannot make this daily itinerary. However, by using fully autonomous vehicles, the charging actions can be performed by the vehicle itself. This means that charging stations don't need to be at the visited location and the EV owners will not care too much about the charging time. The following results demonstrate how our proposed models and algorithms help to implement the optimal charging decision-making under the connected and automated vehicles framework.

Figure 17 provides the energy states when arriving at each location without charging actions. In this figure, the starting location home is not considered. We assume that it has a fully charged battery energy state at home. Number 7 


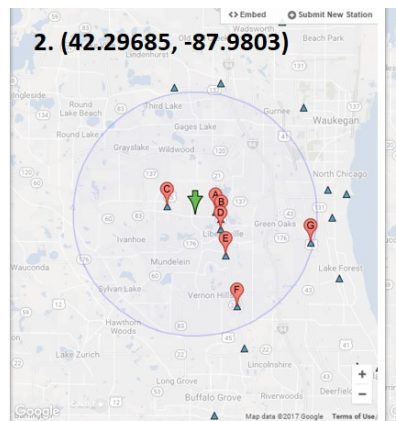

3. $(42.27636,-87.9671)$
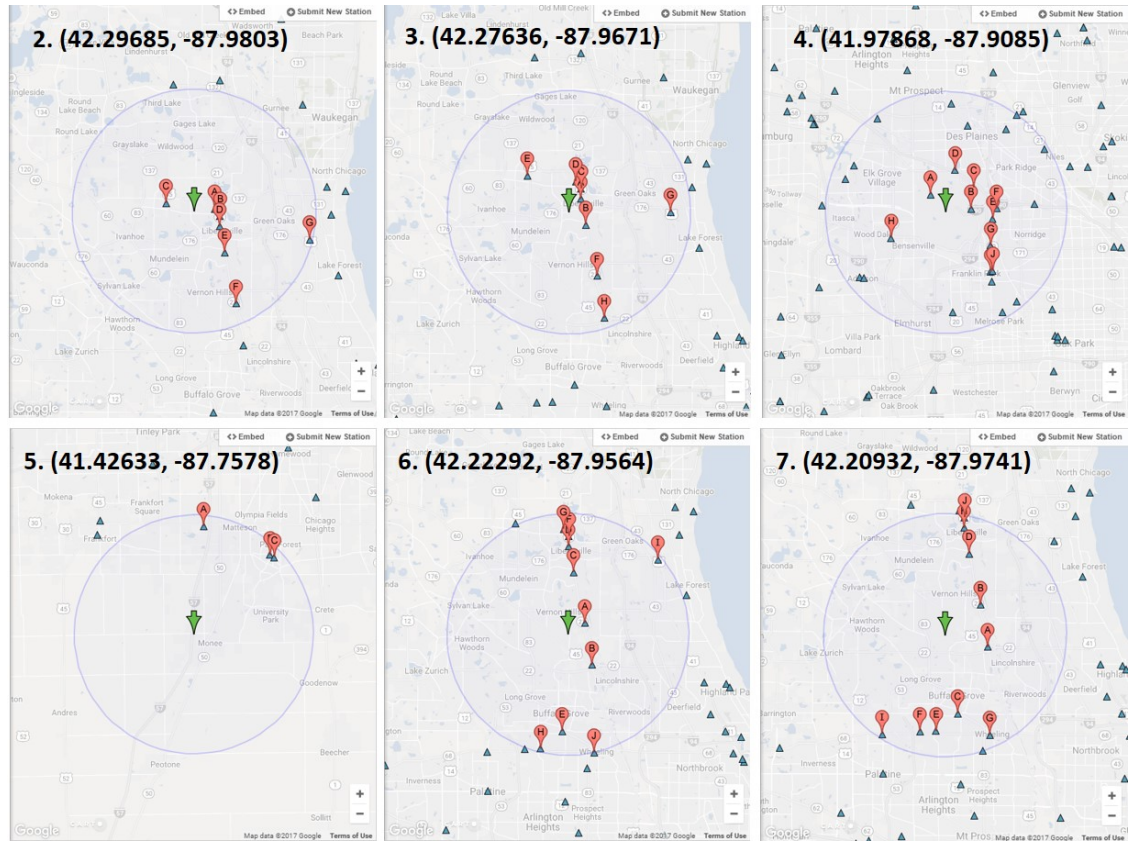

Figure 15: Level 2 charging station around the visited location with 6-mile range
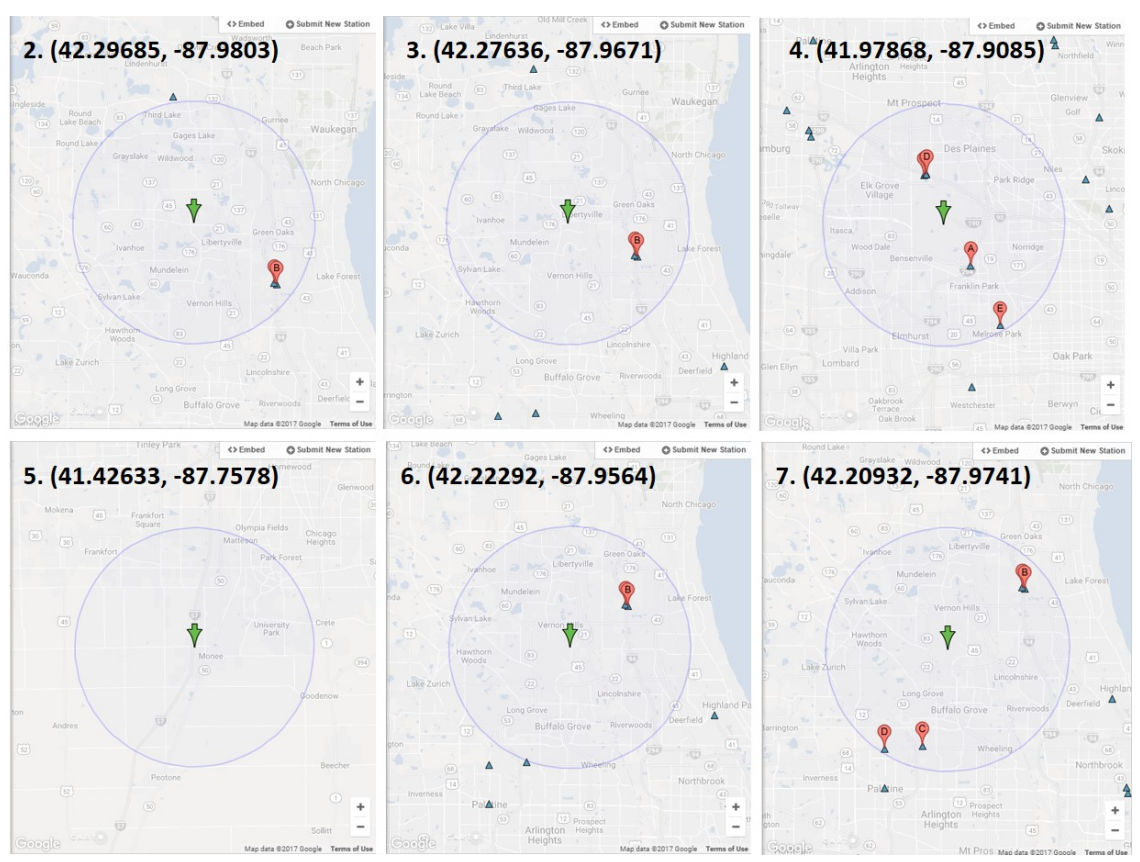

Figure 16: DC fast charging station around the visited location with 6-mile range 


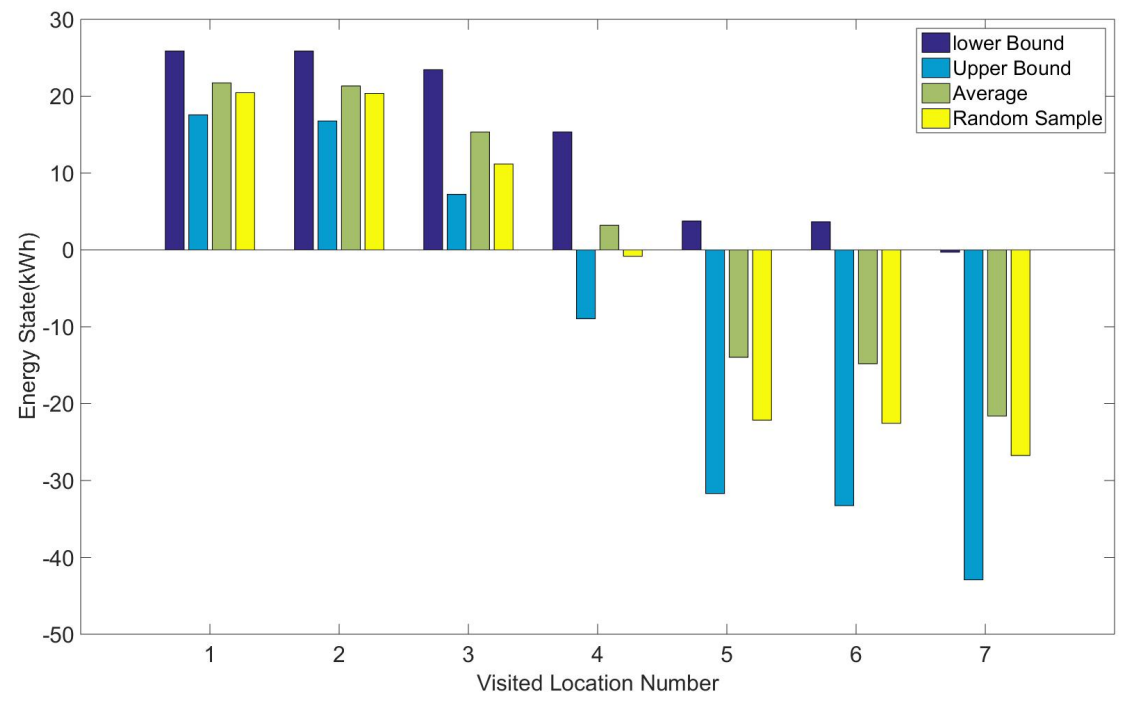

Figure 17: Energy state during the itinerary without charging actions

is used to represent the home for the final destination. The other six locations are the corresponding visited places. A Nissan Leaf with battery capacity of $30 \mathrm{kWh}$ is used in this case study. Figure 17 checks four different situations. According to the proposed stochastic energy cost model, energy cost on each trip segment should be random and we have the algorithm to find the lower bound and upper bound for the energy cost. Then four different situations, i.e. lower bound, upper bound, average and random case, are demonstrated. Without charging action during this itinerary, energy states will be negative after several trips. This means that the charging actions outside home are definitely necessary to complete this daily itinerary with enough energy.

In this case study, predictions of energy cost on all trip segments for the multistage decision-making model can be categorized into two parts: First, the average energy cost information on each trip is utilized by the deterministic decision-making model; Second, the lower and upper bounds of energy cost are used to construct the energy constraint in the robust model, and the random energy cost information are applied into the energy state transition equation for the dynamics of energy state. The nearby charging station information will be fed into both models. According to the distance and travel time from visited location to charging stations, energy costs of charging actions are predicted by the proposed energy consumption prediction model. These information are fed into these two models respectively. Besides these energy cost information, the charging capability of nearby charging stations and also the corresponding charging cost are also necessary to specify the decision-making model. Once all these information are collected and prepared, we can solve both of the de- 


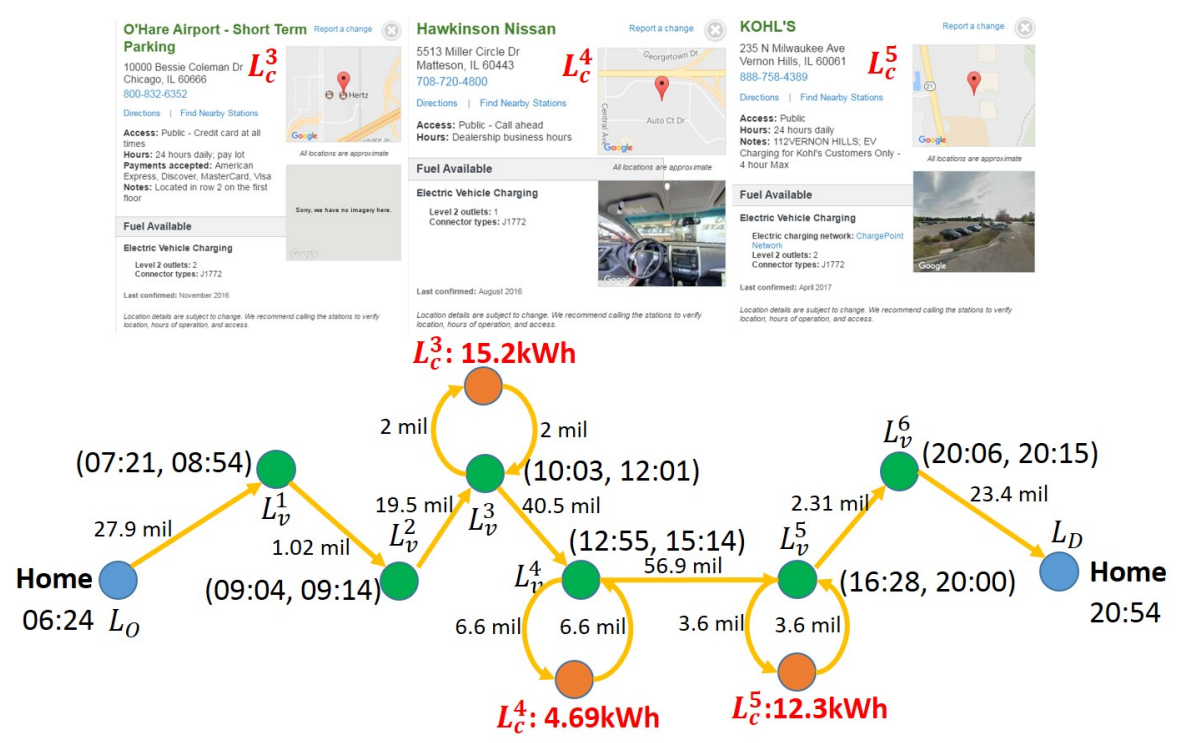

Figure 18: Deterministic Charging Decision Making

terministic and robust decision-making problems to find the optimal charging strategies.

Figure 18 provides the charging strategies by considering the average and deterministic case. Figure 19 demonstrates the charging strategies with the consideration of uncertainties of energy cost. Detailed distance information on each trip segment, arrival and departure time at each visited location are specified. The optimal charging strategies include the amount of charged energy and also the location of selected charging station. For average situation, three charging stations $L_{c}^{3}, L_{c}^{4}$ and $L_{c}^{5}$ nearby $L_{v}^{3}, L_{v}^{4}$ and $L_{v}^{5}$ respectively are selected to preform charging actions. CAEV is charged by $15.2 \mathrm{kWh}, 4.69 \mathrm{kWh}$ and $12.3 \mathrm{kWh}$, respectively. For robust situation, the CAEV also make the charging actions nearby these three locations but using different charging stations. The amount of charged energy for each charging action is also different. They are $9.15 \mathrm{kWh}$, $6.53 \mathrm{kWh}$ and $23.1 \mathrm{kWh}$, respectively. In order to achieve safer charging strategies with consideration of energy cost uncertainties, more overall energy are charged in the robust situation.

Figure 20 provides more detailed comparisons between these two optimal charging strategies. This result illustrates the energy state of CAEV at all visited locations. Here location 7 is home as the destination. We can see that both proposed charging strategies can make sure the energy state of CAEV always positive. This means that a Nissan Leaf with $30 \mathrm{kWh}$ can have enough energy to finish the itinerary by using the proposed charging actions. However, different remaining energies have been derived for these two strategies when they arrive home. It is reasonable that more energy remains for the robust 


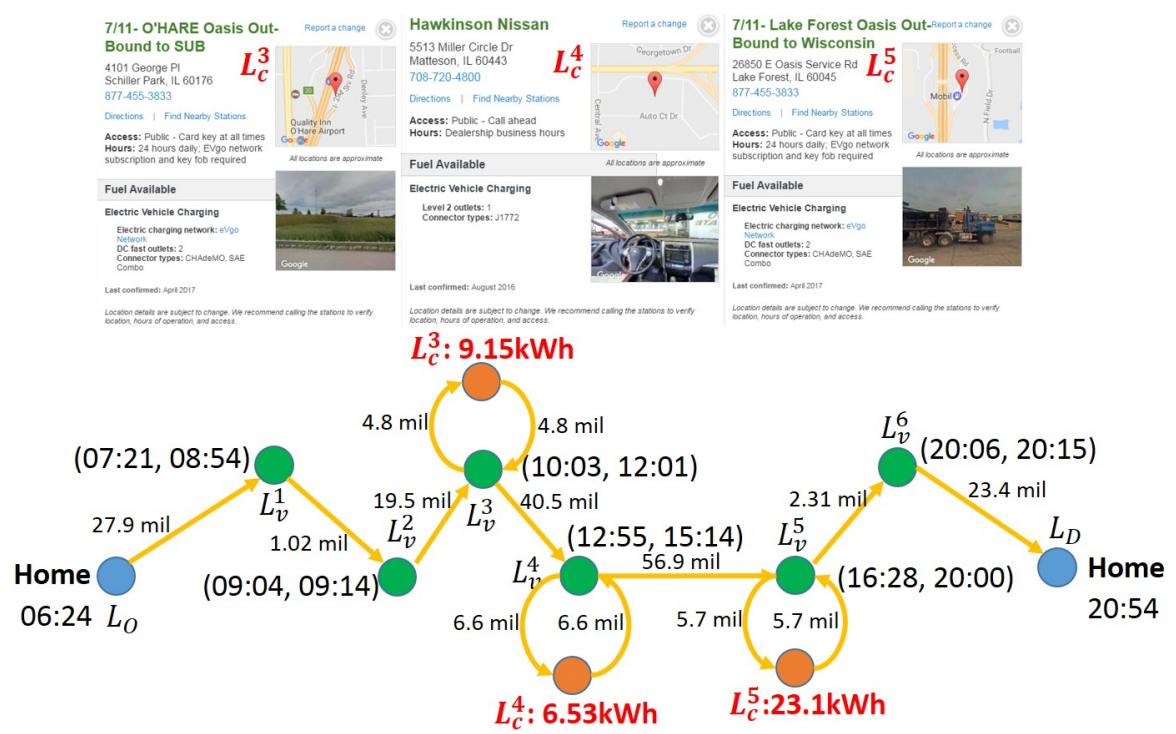

Figure 19: Robust Charging Decision Making

strategy due to the consideration of energy cost uncertainties.

\subsubsection{Case Studies on the Whole Dataset}

In order to show the advantages of the proposed models, more comprehensive studies are performed to simulate various scenarios with a huge of itineraries on the whole dataset. Figure 21 illustrates all visited locations for all itineraries in the whole CRHTI dataset and locations of Level 2 and DC fast charging station in Chicago area from the website of Alternative Fuels Data Center from U.S. Department of Energy (AFDC (2017)). These locations information and the corresponding itinerary information are the input of our case studies. Each valid itinerary has the same detailed information as that in single itinerary case study. In total, 18826 itineraries are fed into the energy consumption and charging decision model for case studies.

Figure 22 illustrates percentages of charging necessity for the investigated itineraries by using the proposed energy consumption model in this paper. Our research in this paper tries to study the charging decision making outside home. It is valuable to see how often it is necessary for charging actions outside home. Results show that most of daily itineraries in Chicago can be finished without charging actions even with small battery initial energy state. However, more and more itineraries will need charging actions by reducing the initial energy state. This means that it is worth designing charging decision algorithm to help the EVs finish their itineraries successfully even with a low initial battery energy state for coming itineraries.

Figure 23 provides the simulation results by using the proposed CAEV charging decision making framework. Results in Figure 23 only includes simulations 


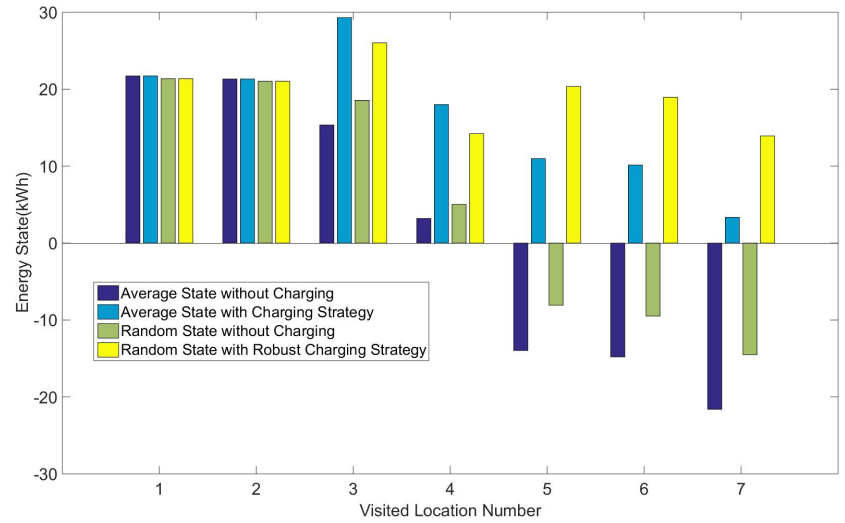

Figure 20: Energy state during the itinerary with charging actions
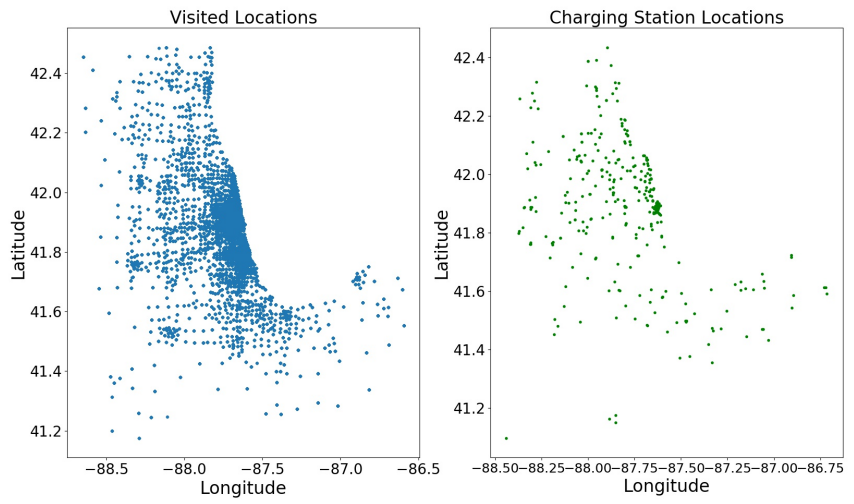

Figure 21: Visited locations distribution in the whole CRHTI and charging station locations distribution (Level 2 and DC fast Charging station) in Chicago 


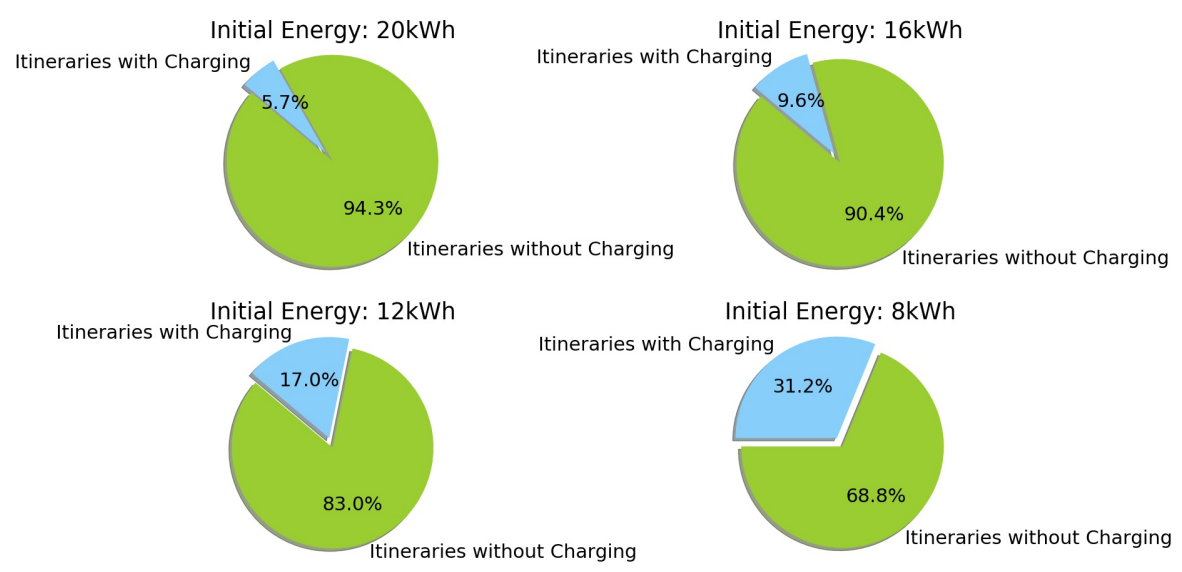

Figure 22: Percentages of charging necessity for all the itineraries with different initial energy states

of itineraries which need charging actions. These itineraries are obtained from calculations under the corresponding battery energy states in Figure 22. The case of $20 k W h$ has 1067 itineraries. The case of $16 k W h$ has 1808 itineraries. The case of $12 k W h$ has 3209 itineraries. The case of $8 k W h$ has 5871 itineraries. Each itinerary is simulated, calculated and checked the same as what has done in the case study of single itinerary. The charging decision making algorithm provides the charging strategies by using the existing charging station network as shown in Figure 21. If the itinerary with the proposed charging strategies can be finished successfully (this means CAEV can always have positive energy during the itinerary), we count this itinerary into the successful part. Therefore, Figure 23 provides the percentages of successful itineraries under different initial battery state conditions and also different available trip prediction information in the decision making model. Here the one-step and two-step predictions are discussed in detail in Section 4.3. It means how much future trip information we can have or predict during the decision making process. For example, decision making model with two-step prediction can have the prediction capability of two trip segments during the itinerary. Results in Figure 23 shows that, under the same initial battery energy state, a decision making model with stronger prediction capability can make more itineraries successful. We can also see that, CAEVs with the proposed charging decision making algorithm can complete most of the trips even at a very low initial battery state, e.g. $8 k W h$, only by using the current existing charging station network. There are still some failed itineraries. This is limited by the long distance of some itineraries and also density of charging stations. This means more charging station are still necessary to make sure all the itineraries can be completed successfully. Percentages of 


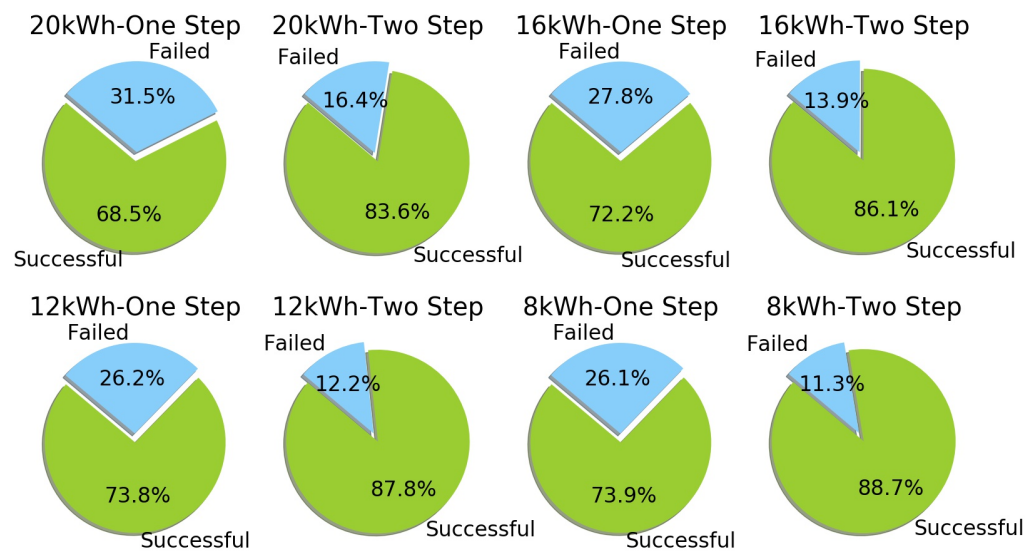

Figure 23: Percentages of successful itineraries for CAEVs under different initial battery energy states and prediction strategies by using current existing charging station network

successful itineraries are smaller when EVs have larger initial energy state. This is due to the smaller size of overall simulated itineraries. Actually the absolute number of successful itineraries is larger, which can be calculated by using the percentage value and the overall itinerary number.

All these case studies in this section demonstrate that the introduced charging decision making algorithm can help CAEVs to reduce the dependency on charging stations. Even only using the current existing charging station network and at low initial battery energy state conditions, most of itineraries can be completed successfully. This means that CAEVs equipped with the introduced charging decision making algorithm can reduce the range anxiety.

\section{Conclusions and Discussions}

Optimal charging decision-making has been studied in this paper under a connected and automated electric vehicles framework for personal usage. A data-driven method based on numerous real-world data has been proposed to construct multi-channel stochastic energy consumption prediction model. The number of channels in this model determines the resolution of energy cost prediction. CAEVs equipped with the introduced real-time updating algorithm have the online learning capability for model evolution based on new obtained real-world data. This can keep the energy consumption model always up-todate for accurate predictions. Based on the energy cost prediction model, both deterministic and robust multistage charging decision-making models are established for optimal strategies during a daily itinerary. They will satisfy different strategy requirements. Robust decision-making model is designed to provide 
safer charging strategies under worst-case considerations of energy cost uncertainties. Simulations and case studies demonstrate the functionality of the proposed methodology. The results also show the potential ability of CAEV to reduce the range anxiety and charging infrastructure dependency.

The energy consumption prediction model in this paper is characterized only by average speed. It is a one-dimensional multi-channel model. This may cause large uncertainties. In the future, high-resolution real-world data of energy consumption under many different conditions will be collected and modeled to reduce the uncertainties in the energy cost prediction by constructing multi-dimensional multi-channel models. High-resolution energy cost prediction models will help to improve many decision-making problems in sustainable transportation, for example the charging strategies in this paper, energy-aware routing strategies, etc.

The charging decision making framework needs to know itinerary information in advance. The itinerary information of the entire day is necessary to achieve the global optimality of charging strategies. However, spatial and temporal fluctuations of itinerary information limit us to achieve this. The introduced prediction process in current framework can release this limitation in some extent, but more powerful prediction technologies and comprehensive optimization models for dynamic itinerary information are necessary for better charging decision making in the future work.

This charging decision making framework for CAEVs aims to minimize the charging costs and time outside home during a daily itinerary without consideration of the charging costs and time at home. This limits the introduced framework to be applied to CAEV users who may not have guaranteed charging infrastructure at home. In the future work, it is valuable to generalize the framework to include not only the charging cost at home but also other types of charging cost, e.g. charging at home and workplace, etc.

Current existing charging infrastructure networks are utilized in this paper. However, charging demand patterns from CAEVs in the future will be different from the current transportation network. The future placement of charging infrastructure should involve different patterns of energy demand from CAEVs. A more promising way should combine charging decision making and energy supply together for CAEVs involved transportation system in order to achieve more sustainable system design and implementation.

\section{Acknowledgement}

This work is performed for the U.S. Department of Energy under Idaho National Laboratory contract number AC07-05ID14517. Funding is provided by the U.S. Department of Energys Vehicle Technologies Office. 


\section{References}

AFDC, 2017. Electric vehicle charging station locations. http://www.afdc.energy.gov/fuels/electricit_locations.html, accessed: 2017$04-20$.

Armaroli, N., Balzani, V., 2011. Towards an electricity-powered world. Energy \& Environmental Science 4 (9), 3193-3222.

Bae, S., Kwasinski, A., 2012. Spatial and temporal model of electric vehicle charging demand. IEEE Transactions on Smart Grid 3 (1), 394-403.

Ben-Tal, A., El Ghaoui, L., Nemirovski, A., 2009. Robust optimization. Princeton University Press.

Bilgin, B., Magne, P., Malysz, P., Yang, Y., Pantelic, V., Preindl, M., Korobkine, A., Jiang, W., Lawford, M., Emadi, A., 2015. Making the case for electrified transportation. IEEE Transactions on Transportation Electrification 1 (1), 4-17.

Birrell, S. A., Wilson, D., Yang, C. P., Dhadyalla, G., Jennings, P., 2015. How driver behaviour and parking alignment affects inductive charging systems for electric vehicles. Transportation Research Part C: Emerging Technologies 58, 721-731.

Chen, T., Zhang, B., Pourbabak, H., Kavousi-Fard, A., Su, W., 2016a. Optimal routing and charging of an electric vehicle fleet for high-efficiency dynamic transit systems. IEEE Transactions on Smart Grid.

Chen, T. D., Kockelman, K. M., Hanna, J. P., 2016b. Operations of a shared, autonomous, electric vehicle fleet: Implications of vehicle \& charging infrastructure decisions. Transportation Research Part A: Policy and Practice 94, 243-254.

Chen, Y., Zhu, L., Gonder, J., Young, S., Walkowicz, K., 2017a. Data-driven fuel consumption estimation: A multivariate adaptive regression spline approach. Transportation Research Part C: Emerging Technologies 83, 134-145.

Chen, Z., Liu, W., Yin, Y., 2017b. Deployment of stationary and dynamic charging infrastructure for electric vehicles along traffic corridors. Transportation Research Part C: Emerging Technologies 77, 185-206.

CMAP, 2007. Chicago regional household travel inventory. http://www.cmap.illinois.gov/data/transportation/travel-tracker-survey, accessed: 2017-04-20.

Corbett, A., Maniaci, R., May 29 2013. Automated electric vehicle charging station. US Patent App. 13/904,811. 
De Cauwer, C., Van Mierlo, J., Coosemans, T., 2015. Energy consumption prediction for electric vehicles based on real-world data. Energies 8 (8), 85738593.

Deflorio, F., Castello, L., 2017. Dynamic charging-while-driving systems for freight delivery services with electric vehicles: Traffic and energy modelling. Transportation Research Part C: Emerging Technologies.

Dong, J., Liu, C., Lin, Z., 2014. Charging infrastructure planning for promoting battery electric vehicles: An activity-based approach using multiday travel data. Transportation Research Part C: Emerging Technologies 38, 44-55.

Eberle, U., Von Helmolt, R., 2010. Sustainable transportation based on electric vehicle concepts: a brief overview. Energy \& Environmental Science 3 (6), 689-699.

electrek, 2017. Gm is increasing its self-driving chevy bolt ev test fleet to 300 vehicles. https://electrek.co/2017/04/17/gm-self-driving-chevy-bolt-evtest-fleet/, accessed: 2017-04-20.

Fagnant, D. J., Kockelman, K. M., 2014. The travel and environmental implications of shared autonomous vehicles, using agent-based model scenarios. Transportation Research Part C: Emerging Technologies 40, 1-13.

Fiori, C., Ahn, K., Rakha, H. A., 2016. Power-based electric vehicle energy consumption model: Model development and validation. Applied Energy 168, $257-268$.

Frade, I., Ribeiro, A., Gonçalves, G., Antunes, A., 2011. Optimal location of charging stations for electric vehicles in a neighborhood in lisbon, portugal. Transportation research record: journal of the transportation research board (2252), 91-98.

Fuller, M., 2016. Wireless charging in california: Range, recharge, and vehicle electrification. Transportation Research Part C: Emerging Technologies 67, 343-356.

Genikomsakis, K. N., Mitrentsis, G., 2017. A computationally efficient simulation model for estimating energy consumption of electric vehicles in the context of route planning applications. Transportation Research Part D: Transport and Environment 50, 98-118.

Ghamami, M., Zockaie, A., Nie, Y. M., 2016. A general corridor model for designing plug-in electric vehicle charging infrastructure to support intercity travel. Transportation Research Part C: Emerging Technologies 68, 389-402.

Giménez-Gaydou, D. A., Ribeiro, A. S., Gutiérrez, J., Antunes, A. P., 2016. Optimal location of battery electric vehicle charging stations in urban areas: A new approach. International Journal of Sustainable Transportation 10 (5), 393-405. 
He, F., Yin, Y., Zhou, J., 2015. Deploying public charging stations for electric vehicles on urban road networks. Transportation Research Part C: Emerging Technologies 60, 227-240.

He, S. Y., Kuo, Y.-H., Wu, D., 2016. Incorporating institutional and spatial factors in the selection of the optimal locations of public electric vehicle charging facilities: A case study of beijing, china. Transportation Research Part C: Emerging Technologies 67, 131-148.

Hu, J., You, S., Lind, M., Ostergaard, J., 2014. Coordinated charging of electric vehicles for congestion prevention in the distribution grid. IEEE Transactions on Smart Grid 5 (2), 703-711.

INL, 2013. The ev project - plug-in electric vehicle charging infrastructure demonstration. https://avt.inl.gov/project-type/ev-project, accessed: 201704-20.

Latinopoulos, C., Sivakumar, A., Polak, J., 2017. Response of electric vehicle drivers to dynamic pricing of parking and charging services: Risky choice in early reservations. Transportation Research Part C: Emerging Technologies $80,175-189$.

Li, S., Huang, Y., Mason, S. J., 2016. A multi-period optimization model for the deployment of public electric vehicle charging stations on network. Transportation Research Part C: Emerging Technologies 65, 128-143.

Marmaras, C., Xydas, E., Cipcigan, L., 2017. Simulation of electric vehicle driver behaviour in road transport and electric power networks. Transportation Research Part C: Emerging Technologies 80, 239-256.

Ondruska, P., Posner, I., 2014. Probabilistic attainability maps: Efficiently predicting driver-specific electric vehicle range. In: Intelligent Vehicles Symposium Proceedings, 2014 IEEE. IEEE, pp. 1169-1174.

Pourazarm, S., Cassandras, C. G., Wang, T., 2016. Optimal routing and charging of energy-limited vehicles in traffic networks. International Journal of Robust and Nonlinear Control 26 (6), 1325-1350.

Prins, R., Hurlbrink, R., Winslow, L., 2013. Electric vehicle energy usage modelling and measurement. International Journal of Modern Engineering 13 (1), $5-12$.

Riemann, R., Wang, D. Z., Busch, F., 2015. Optimal location of wireless charging facilities for electric vehicles: flow-capturing location model with stochastic user equilibrium. Transportation Research Part C: Emerging Technologies $58,1-12$.

Sioshansi, R., Denholm, P., 2009. Emissions impacts and benefits of plug-in hybrid electric vehicles and vehicle-to-grid services. Environmental science \& technology 43 (4), 1199-1204. 
Smart, J., Schey, S., 2012. Battery electric vehicle driving and charging behavior observed early in the ev project. SAE International Journal of Alternative Powertrains 1 (2012-01-0199), 27-33.

Sundstrom, O., Binding, C., 2012. Flexible charging optimization for electric vehicles considering distribution grid constraints. IEEE Transactions on Smart Grid 3 (1), 26-37.

Tesla, 2016. All tesla cars being produced now have full self-driving hardware. https://www.tesla.com/blog/all-tesla-cars-being-produced-now-have-fullself-driving-hardware, accessed: 2017-04-20.

Tesla, 2017. Tesla patent shows new way to automated high-speed charging with external cooling. https://electrek.co/2017/04/22/tesla-patentautomate-charging/\#more-42432, accessed: 2017-04-25.

Tu, W., Li, Q., Fang, Z., Shaw, S.-l., Zhou, B., Chang, X., 2016. Optimizing the locations of electric taxi charging stations: A spatial-temporal demand coverage approach. Transportation Research Part C: Emerging Technologies $65,172-189$.

UITP, 2017. Autonomous vehicles: a potential game changer for urban mobility. http://www.uitp.org/sites/default/files/cck-focus-papersfiles/PolicyBrief-Autonomous-Vehicles-LQ-20160116.pdf, accessed: 2017-0824.

Wang, Q., Liu, X., Du, J., Kong, F., 2016. Smart charging for electric vehicles: A survey from the algorithmic perspective. IEEE Communications Surveys \& Tutorials 18 (2), 1500-1517.

Welford, B., 1962. Note on a method for calculating corrected sums of squares and products. Technometrics 4 (3), 419-420.

Xylia, M., Leduc, S., Patrizio, P., Kraxner, F., Silveira, S., 2017. Locating charging infrastructure for electric buses in stockholm. Transportation Research Part C: Emerging Technologies 78, 183-200.

Yang, J., Dong, J., Hu, L., 2017. A data-driven optimization-based approach for siting and sizing of electric taxi charging stations. Transportation Research Part C: Emerging Technologies 77, 462-477.

Yang, Y., Yao, E., Yang, Z., Zhang, R., 2016. Modeling the charging and route choice behavior of bev drivers. Transportation Research Part C: Emerging Technologies 65, 190-204.

Yi, Z., Bauer, P. H., 2016a. Optimization models for placement of an energyaware electric vehicle charging infrastructure. Transportation Research Part E: Logistics and Transportation Review 91, 227-244. 
Yi, Z., Bauer, P. H., 2016b. Spatiotemporal energy demand models for electric vehicles. IEEE Transactions on Vehicular Technology 65 (3), 1030-1042.

Yi, Z., Bauer, P. H., 2017a. Adaptive multi-resolution energy consumption prediction for electric vehicles. IEEE Transactions on Vehicular Technology.

Yi, Z., Bauer, P. H., 2017b. Effects of environmental factors on electric vehicle energy consumption: A sensitivity analysis. IET Electrical Systems in Transportation 7 (1), 3-13.

Zhang, R., Yao, E., 2015. Electric vehicles energy consumption estimation with real driving condition data. Transportation Research Part D: Transport and Environment 41, 177-187. 